\title{
LOGIC FOR EXACT ENTAILMENT
}

\author{
KIT FINE AND MARK JAGO
}

\begin{abstract}
An exact truthmaker for $A$ is a state which, as well as guaranteeing $A$ 's truth, is wholly relevant to it. States with parts irrelevant to whether $A$ is true $\operatorname{do}$ not count as exact truthmakers for $A$. Giving semantics in this way produces a very unusual consequence relation, on which conjunctions do not entail their conjuncts. This feature makes the resulting logic highly unusual. In this paper, we set out formal semantics for exact truthmaking and characterise the resulting notion of entailment, showing that it is compact and decidable. We then investigate the effect of various restrictions on the semantics. We also formulate a sequent-style proof system for exact entailment and give soundness and completeness results.
\end{abstract}

§1. Introduction. There are a number of different ways in which a state or fact can be said to be a truthmaker for a sentence. It can simply necessitate the sentence; or it can necessitate the sentence while being partially relevant to its truth; or it can necessitate the sentence while being wholly relevant to its truth. Our interest in the present paper is in the third, exact, mode of truthmaking.

The notion of exact truthmaking has lately been applied to a number of different topics in philosophy and linguistics [Fine, 2017] and it has natural affinities with the notion of ground in metaphysics. Given the significance of the notion, it is therefore of interest to determine the logic to which it gives rise. If truthmaking is taken to be wholly relevant, as on the exact model, rather than simply a matter of necessitation, as on the classical model, then what will be the resulting notion of entailment and how will it behave?

It turns out that this question is not altogether unambiguous. When we have the entailment of one formula by another, we may simply say that the one formula $A$ exactly entails the other $C$ when every exact truthmaker for $A$ is an exact truthmaker for $C$. But when several formulas $A_{1}, A_{2}, \ldots$ are taken to entail another $C$, there are two different ways in which we might proceed. We might say, on a distributive reading, that every truthmaker for each of $A_{1}, A_{2}, \ldots$ should be a truthmaker for $C$; or we might say, on a collective reading, that every truthmaker for the conjunction $A_{1} \wedge A_{2} \wedge \cdots$ of $A_{1}, A_{2}, \ldots$ should be a truthmaker

1991 Mathematics Subject Classification. 03B47, 03B60.

Key words and phrases. Truthmaking, exact entailment, non-classical logic, semantics, mereology, sequent calculus.

The paper arose in the following way. Mark Jago initially wrote a paper on the topic. This contained the characterisation theorem, but proved in a somewhat complicated way. He later learned of Kit Fine's related unpublished work on the collective notion of exact entailment; and they decided to join forces, thereby extending some of their previous results and simplifying some of the proofs. 
for $C$. These two notions of entailment will coincide in the classical case but not in the exact case. For $A, B$ will exactly entail $A$ in the distributive sense of entailment but not in the collective sense, since there is, in general, no guarantee that an exact truthmaker for $A \wedge B$ will be an exact truthmaker for $A$. Since $A$ entails $A$ (in either sense), the collective sense of entailment, in contrast to the distributive sense, will not be monotonic.

The present paper focuses on the first, distributive, sense of exact entailment and is largely concerned with the special problems which arise when the same state is taken to be a truthmaker for different formulas. These problems are partly proof theoretic in character, since we are no longer entitled to infer from the fact that $A, B$ exactly entails $C$ that $A \wedge B$ exactly entails $C$. But they are also partly semantic, since it is not altogether clear what is required of a state when it is taken to be a truthmaker for two different formulas, such as $A \wedge B$ and $B \wedge C$.

One philosophical motivation for this distributive, truthmaker-sharing notion of entailment comes from multiple realizability, as in the philosophy of mind. Suppose mental statement $M$ has both physical and non-physical possible realizers, but we want to focus on its physical realizers. These are the states which are truthmakers for both $M$ and the statement $P$ that there exist physical states (or, if you prefer, the infinite disjunction of all physical statements). We can conceptualise $P$ as a condition on realizers for $M$ and investigate the exact consequences of $P$ and $M$, taken as distributive premises. Similar examples arise with determinable properties and conditions on their determinates.

A different kind of use arises in the investigation of trivial truth, where a truth is trivial in the sense that it requires nothing substantive to make it true (or, as we prefer to say, when it is made true by the null state). It is plausible, for example, that $A \rightarrow A$ is trivial in this sense, whereas $A \wedge \neg A$ is not. For the latter in general requires something substantive to make it true: a substantive truthmaker either for $A$ or for $\neg A$; whereas $A \rightarrow A$ requires not even that. We can introduce a constant $T$, by definition made true by every state, and investigate the consequences of distributive premises $A$ and T. If $\perp$ (by definition made true by no state) is not a consequence, then $A$ is a trivial truth in the intended sense. (We do not investigate $\rightarrow, T$, or $\perp$ further in this paper.)

However, our interest in the distributive notion of entailment is as much due to logical as to philosophical curiosity. The single-premise notion of exact entailment is already of great interest and so the question arises as to how should it be extended to the multi-premise case. If one holds that the logical notion of entailment should be monotonic in its premises, then the distributive notion presents itself as the obvious choice.

A rough outline of the paper is as follows. $\S 2$ presents the basic semantic framework and defines the relevant notions of exact truthmaking and exact entailment. $\S 3$ defines 'selections', the syntactic counterparts of truthmakers, and introduces the corresponding notion of a canonical model. $\S 4$ is central to the whole paper; it gives a characterisation of exact entailment in terms of selections. Of perhaps special interest is the method of proof, which depends upon defining a congruence on a canonical model, in something like the same way in which 'filtrations' are defined in modal logic. $\S 5$ applies the characterisation result to 
establish decidability and compactness. $\S 6$ and $\S 7$ consider various further constraints that might be imposed on our models, and investigate the question of whether these further constraints result in any changes to the logic. $\S 8$ presents a sequent calculus, which is somewhat more complicated than usual, since $A, B$ on the left of a sequent cannot be interpreted conjunctively. We therefore require two 'structural' connectives, one interpreted distributively and the other conjunctively. Finally, $\S 9$ establishes soundness and completeness for the system, and the eliminability of (CUT).

§2. Exact Truthmaker Semantics. It is characteristic of exact truthmaking that truthmakers for a conjunction are not in general truthmakers for its conjuncts. A truthmaker for $A \wedge B$ is the fusion of a truthmaker for $A$ and a truthmaker for $B$. We here have in mind the usual mereological notion of fusion, according to which a fusion is a structureless whole formed from its component parts [Varzi, 2016]. Writing $s \sqcup t$ for the fusion of $s$ and $t$, we thus have:

$s$ truthmakes $A \wedge B$ if and only if, for some $t, u$ where $t$ truthmakes $A$ and $u$ truthmakes $B, s=t \sqcup u$.

This is, in essence, van Fraassen's clause for conjunction [van Fraassen, 1969, 484].

What should we say about disjunctions? A disjunction is made true by whatever makes at least one of its disjuncts true, so we might say:

$s$ truthmakes $A \vee B$ if and only if either $s$ truthmakes $A$ or $s$ truthmakes $B$.

However, this seems to exclude a further way of making a disjunction true, namely, by being made up of parts which individually make the disjuncts true. After all, the state of affairs that Anna is walking and Bec is sitting is wholly relevant to the truth of 'Anna is walking or Bec is sitting', even if it is overkill. So we may instead say:

$s$ truthmakes $A \vee B$ if and only if $s$ truthmakes $A, s$ truthmakes $B$, or $s=t \sqcup u$ for some truthmakers $t$ for $A$ and $u$ for $B$.

On the latter conception (though not on the former), truthmakers for $A \wedge B$ are also truthmakers for $A \vee B$, and so (if we define entailment as truthmakerpreservation) $A \wedge B$ will entail $A \vee B$. To keep things simple, we will adopt the latter clause for disjunctions here. Nevertheless, all of the results that follow will go through if we adopt the former, under a suitable modification of definition 3.2 in $\S 3$.

We have said what the truthmakers for conjunctions and disjunctions are, so we now need to say something about their falsemakers too. But this is straightforward. A falsemaker for $A \wedge B$ is something which falsemakes $A$, or $B$, or else is the fusion of such falsemakers; a falsemaker for $A \vee B$ is a fusion of a falsemaker for $A$ and a falsemaker for $B$; and a falsemaker for $\neg A$ is a truthmaker for $A$ (while a truthmaker for $\neg A$ is a falsemaker for $A$ ).

Having given an overview of our semantics, it is time to make it more precise.

Definition 2.1 (Syntax). Let $\mathcal{P}$ be a set of sentence letters. The language $\mathcal{L}$ is then the smallest set containing $\mathcal{P}$ closed under 1-place $\neg$ and 2-place $\wedge$ and 
$\vee . \Lambda$ is the set of literals over $\mathcal{P}$, i.e. $\mathcal{P} \cup\{\neg p: p \in \mathcal{P}\}$. Let $\leqslant_{\mathcal{L}}$ be any total order on $\mathcal{L}$. We define $\bigwedge\left\{A_{1}, \ldots, A_{n}\right\}=\left(A_{1} \wedge\left(A_{2} \wedge \cdots \wedge A_{n}\right) \cdots\right)$, where $A_{i} \leqslant_{\mathcal{L}} A_{j}$ iff $i \leqslant j$. (We shall see below that $\wedge$ is commutative and associative, and so brackets and order of conjuncts is semantically irrelevant.)

We take $\mathcal{P}$ (and hence $\mathcal{L}$ and $\Lambda$ ) to be fixed throughout.

Definition 2.2 (Frames and models). Frames are structures $\langle S$, $\sqsubseteq\rangle$ where $S$ is a set of entities (states) and $\sqsubseteq$ is a partial order (reflexive, transitive, and antisymmetric), such that each pair $s, t \in S$ has a least upper bound in $S$, denoted $s \sqcup t$. An exact truthmaking model is a structure $\left\langle S, \sqsubseteq,|\cdot|^{+},|\cdot|^{-}\right\rangle$where $\langle S$, 드 is a frame and $|\cdot|^{+}$and $|\cdot|^{-}$are valuation functions from sentence letters $\mathcal{P}$ to $2^{S}$, closed under $\sqcup$ : if $s, t \in|p|^{+}$then $s \sqcup t \in|p|^{+}$, and similarly for $|p|^{-}$.

DEFINITION 2.3 (Exact truthmaking and falsemaking). Given a exact truthmaking model $M=\left\langle S, \sqsubseteq,|\cdot|^{+},|\cdot|^{-}\right\rangle$(which we leave implicit), exact truthmaking $\Vdash$ and exact falsemaking $\dashv$ relations are then defined by double recursion as follows:

$$
\begin{array}{rcl}
s \Vdash p & \text { iff } & s \in|p|^{+} \\
s \dashv p & \text { iff } & s \in|p|^{-} \\
s \Vdash \neg A & \text { iff } & s \dashv A \\
s \dashv \neg A & \text { iff } & s \Vdash A \\
s \Vdash A \wedge B & \text { iff } & \exists t u(s=t \sqcup u \& t \Vdash A \& u \Vdash B) \\
s \dashv A \wedge B & \text { iff } & s \dashv A \text { or } s \dashv B \text { or } \\
& \exists t u(s=t \sqcup u \& t \dashv A \& u \dashv B) \\
s \Vdash A \vee B & \text { iff } \quad & s \Vdash A \text { or } s \Vdash B \text { or } \\
& \quad \exists t u(s=t \sqcup u \& t \Vdash A \& u \Vdash B) \\
s \dashv A \vee B & \text { iff } \quad \exists t u(s=t \sqcup u \& t \dashv A \& u \dashv B)
\end{array}
$$

Given a set of formulas $\Gamma$, we define $s \Vdash \Gamma(s \dashv \Gamma)$ just in case $s \Vdash B(s \dashv B)$ for each $B \in \Gamma$. We extend the $|\cdot|^{+}$and $|\cdot|^{-}$notation to all formulas $A$ by setting $|A|^{+}=\{s \mid s \Vdash A\}$ and $|A|^{-}=\{s \mid s \dashv A\}$.

Given this semantics, no formula is valid (in the sense of being true at all states in all models), and any set of formulas is satisfiable (just consider a state which both truthmakes and falsemakes every sentence letter $p$ ). We characterise our logic in terms of the entailments it supports. As usual, we treat entailment as the preservation of truth from premises to conclusion. But in so doing, how should we conceptualise our premises? As mentioned in $\S 1$, we could treat them collectively, so that entailment amounts to preservation of truth from the conjoined premises to the conclusion. Alternatively, we could treat our premises distributively, so that entailment amounts to preservation of truth from each individual premise to the conclusion. These notions coincide on single-premise entailments, but are not equivalent when we have multiple premises.

When there are no further restrictions on the models we use, the conjunctive approach will give us a nonmonotonic entailment relation. $A$ will conjunctivelyentail $A$, but $\{A, B\}$ will not conjunctively-entail $A$ (since $A \wedge B$ does not, in 
general, conjunctively-entail $A$.) Fine investigates this conjunctive notion of entailment in combination with various restrictions on models [Fine, 2016]. Accordingly, we shall not say any more about it here.

We shall focus on the distributive notion of entailment, abbreviated as ' $\models$ ', which we define as follows:

Definition 2.4 (Exact Truthmaking Entailment). $\Gamma \models A$ iff, for any model $M$ and any state $s$ of $M, M, s \Vdash A$ whenever $M, s \Vdash \Gamma$.

Exact equivalence is defined as two-way exact entailment. As the reader can easily check, exact entailment satisfies the following equivalences and entailments:

$$
\begin{array}{rlrl}
A \wedge B & =B \wedge A & A \vee B & =B \vee A \\
A \wedge(B \wedge C) & =\models(A \wedge B) \wedge C & A \vee(B \vee C) & =(A \vee B) \vee C \\
A \wedge(B \vee C) & =\models(A \wedge B) \vee(A \wedge C) & A \vee(B \wedge C) \models(A \vee B) \wedge(A \vee C) \\
A \wedge A & =A & A \vee A \Rightarrow \models A \\
\neg(A \wedge B) & =\neg A \vee \neg B & \neg(A \vee B) & =\neg A \wedge \neg B \\
A, B & \models A \wedge B & A & \models A \vee B \\
\neg \neg A & =A &
\end{array}
$$

Note that distribution of $\vee$ over $\wedge$ is not an equivalence, for $(p \vee q) \wedge(p \vee r) \not=$ $p \vee(q \wedge r)$. To see this, take the model:

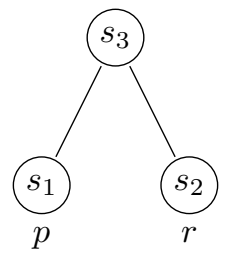

In this model, $s_{1} \Vdash p \vee q$ and $s_{2} \Vdash p \vee r$, hence $s_{3} \Vdash(p \vee q) \wedge(p \vee r)$, but $s_{3} \Vdash \forall \vee(q \wedge r)$. Given the failure of $\vee$ to distribute over $\wedge$, our semantics does not give rise to a $\wedge, \vee$-distributive lattice. This is precisely what makes reasoning about the semantics more difficult (and more interesting) than would otherwise be the case.

Note also that, since $\wedge$ is commutative and associative, it is harmless to write $\bigwedge\left\{A_{1}, \ldots, A_{n}\right\}$ as $A_{1} \wedge \cdots \wedge A_{n}$, and to ignore the ordering $\leqslant_{\mathcal{L}}$.

We now turn to some results characterising the exact semantics, beginning in the next section and culminating, in $\S 4$, with a characterisation theorem for exact truthmaker entailment.

§3. Syntactic Selections and the Canonical Model. In this section, we describe a way to extract from a sentence $A$ a description of the definite ways a truthmaker for $A$ should be. These descriptions take the form of a set of literals. Intuitively, a description $\left\{\lambda_{1}, \ldots, \lambda_{n}\right\}$ describes a state $s$ which is the fusion of states $s_{1}, \ldots, s_{n}$, where each $s_{i}$ truthmakes literal $\lambda_{i}$. We will call such a description, qua set of literals, a selection from $A$. 
As selections provide us with a syntactic means to reason about our semantics, it is natural to begin our discussion by constructing a corresponding canonical model.

Definition 3.1 (Canonical Model). Let $\mathfrak{M}=\left\langle\mathfrak{S}^{\mathfrak{M}}, \sqsubseteq^{\mathfrak{M}},|\cdot|^{+\mathfrak{M}},|\cdot|^{-\mathfrak{M}}\right\rangle$ where:

- $\mathfrak{S}^{\mathfrak{M}}=2^{\Lambda}$, the set of all sets of literals;

- $\subseteq^{\mathfrak{M}}$ is $\subseteq$ restricted to $\mathfrak{S}^{\mathfrak{M}}$;

- $|p|^{+\mathfrak{M}}=\{\{p\}\}$;

- $|p|^{-\mathfrak{M}}=\{\{\neg p\}\}$.

$\mathfrak{M}$ is clearly a genuine model.

Now we can define our notion of a selection from $A$.

Definition 3.2 (Selections). Given a formula $A$, a set of literals $x$ is a selection from $A$ just in case $\mathfrak{M}, x \Vdash A$ (where $\mathfrak{M}$ is the canonical model). The set of all selections from $A, \mathcal{S} A$, is $\{x \mid \mathfrak{M}, x \Vdash A\}$. We extend the terminology to sets of sentences $\Gamma$ : a selection from $\Gamma$ contains one selection from each $A \in \Gamma$. We define the set of all selections from $\Gamma, \mathcal{S} \Gamma$, as follows. When $\Gamma=\left\{A_{1}, A_{2}, \ldots\right\}$ for distinct $A_{1}, A_{2}, \ldots, \mathcal{S} \Gamma$ is the collection of all sets $\left\{x_{1}, x_{2}, \ldots\right\}$, for each $x_{i}$ a selection from $A_{i}$.

We note an equivalent characterisation of $\mathcal{S} A$ :

$$
\begin{aligned}
\mathcal{S} p & =\{\{p\}\} \text { and } \mathcal{S} \neg p=\{\{\neg p\}\} \\
\mathcal{S}(A \wedge B) & =\{x \cup y \mid x \in \mathcal{S} A, y \in \mathcal{S} B\} \\
\mathcal{S} \neg(A \wedge B) & =\mathcal{S} \neg A \cup \mathcal{S} \neg B \cup \mathcal{S}(\neg A \wedge \neg B) \\
\mathcal{S}(A \vee B) & =\mathcal{S} A \cup \mathcal{S} B \cup \mathcal{S}(A \wedge B) \\
\mathcal{S} \neg(A \vee B) & =\{x \cup y \mid x \in \mathcal{S} \neg A, y \in \mathcal{S} \neg B\} \\
\mathcal{S} \neg \neg A & =\mathcal{S} A
\end{aligned}
$$

This presentation brings out the similarity between our notion of a selection and van Fraassen's definition [van Fraassen, 1969, 484] of truthmaking and falsemaking sets.

The following lemmas show that selections are closed under union and, more importantly, establish the relationship between $A$ 's selections and the states that truthmake $A$.

Lemma 3.3. For any model $M$ and states $s, u$ in $M$, if $s \Vdash A$ and $u \Vdash A$ then $s \sqcup u \Vdash A$.

Proof. By induction on $A$. Assume $s \Vdash A$ and $u \Vdash A$. The base case holds by definition 2.2. So assume the result holds for all $B$ less complex than $A$ and consider these cases.

- $A:=B \wedge C$. Then $s=s_{1} \sqcup s_{2}, u=u_{1} \sqcup u_{2}$, where $s_{1} \Vdash B, s_{2} \Vdash C$, $u_{1} \Vdash B$, and $u_{2} \Vdash C$. By hypothesis, $s_{1} \sqcup u_{1} \Vdash B$ and $s_{2} \sqcup u_{2} \Vdash C$, so $s_{1} \sqcup u_{1} \sqcup s_{2} \sqcup u_{2} \Vdash B \wedge C$, hence $s \sqcup u \Vdash B \wedge C$.

- $A:=B \vee C$. Then (i) $s \Vdash B$, (ii) $s \Vdash C$, or (iii) $s=s_{1} \sqcup s_{2}$ where $s_{1} \Vdash B$ and $s_{2} \Vdash C$; and (iv) $u \Vdash B$, (v) $u \Vdash C$, or (vi) $u=u_{1} \sqcup u_{2}$ where $u_{1} \Vdash B$ and $u_{2} \Vdash C$. If (i) \& (iv), then by hypothesis, $s \sqcup u \Vdash B$ and so $s \sqcup u \Vdash B \vee C$. If 
(i) \& (v), then by definition $s \sqcup u \Vdash B \vee C$. If (i) \& (vi), then by hypothesis, $s \sqcup u_{1} \Vdash B$, and so $\left(s_{1} \sqcup u_{1}\right) \sqcup u_{2} \Vdash B \vee C$, hence $s \sqcup u \Vdash B \vee C$. The (ii) \& (iv), (ii) \& (v), (ii) \& (vi), (iii) \& (iv), and (iii) \& (v) cases are similar. Finally, if (iii) and (vi), then by hypothesis, $s_{1} \sqcup u_{1} \Vdash B$ and $s_{2} \sqcup u_{2} \Vdash C$, so $\left(s_{1} \sqcup u_{1}\right) \sqcup\left(s_{2} \sqcup u_{2}\right) \Vdash B \vee C$ and hence $s \sqcup u \Vdash B \vee C$.

- The $A:=\neg(A \wedge B)$ and $A:=\neg(A \vee B)$ cases are similar, and the $A:=\neg \neg B$ case is trivial.

Corollary 3.4. If $x, y \in \mathcal{S} A$ then $x \cup y \in \mathcal{S} A$.

Proof. Suppose $x, y \in \mathcal{S} A$. Then by definition $3.2, \mathfrak{M}, x \Vdash A$ and $\mathfrak{M}, y \Vdash A$ (where $\mathfrak{M}$ is the canonical model). By lemma 3.3 , we have $\mathfrak{M}, x \sqcup y \Vdash A$. Since $x \sqcup y=x \cup y$ in $\mathfrak{M}$, we have $\mathfrak{M}, x \cup y \Vdash A$ and hence $x \cup y \in \mathcal{S} A$.

Lemma 3.5 (Selection lemma). For any formula $A$, model $M$ and state $s$ in $M$ :

$$
s \Vdash A \text { iff, for some } x \in \mathcal{S} A, s \Vdash \bigwedge x .
$$

Proof, Left-To-RIght. The proof is by induction on the complexity of $A$. Assume $s \Vdash A$. If $A$ is a literal $p$ or $\neg p$ then $\mathcal{S} A$ is either $\{\{p\}\}$ or $\{\{\neg p\}\}$. So for any $x \in \mathcal{S} A, \bigwedge x=A$ and the result holds. Now assume that the result holds for all $B$ less complex than $A$, and consider these cases:

- If $A:=A_{1} \wedge A_{2}$ then $s=s_{1} \sqcup s_{2}$ where $s_{1} \Vdash A_{1}$ and $s_{2} \Vdash A_{2}$. By hypothesis, there are $x \in \mathcal{S} A_{1}$ and $y \in \mathcal{S} A_{2}$ such that $s_{1} \Vdash \bigwedge x$ and $s_{2} \Vdash \wedge y$. So $s \Vdash(\bigwedge x) \wedge(\bigwedge y)$ and hence $s \Vdash \bigwedge(x \cup y)$. Since $x \cup y \in \mathcal{S} A$ (corollary 3.4), the result holds.

- If $A:=A_{1} \vee A_{2}$ then either (i) $s \Vdash A_{1}$, (ii) $s \Vdash A_{2}$ or (iii) $s=s_{1} \sqcup s_{2}$ where $s_{1} \Vdash A_{1}$ and $s_{2} \Vdash A_{2}$. If (i) then, by hypothesis, there is some $x \in \mathcal{S} A_{1}$ such that $s \Vdash \bigwedge x$. So by definition, $x \in \mathcal{S}\left(A_{1} \vee A_{2}\right)$. Case (ii) is similar. If (iii) then by hypothesis there are $x_{1} \in \mathcal{S} A_{1}$ and $x_{2} \in \mathcal{S} A_{2}$ such that $s_{1} \Vdash \bigwedge x_{1}$ and $s_{2} \Vdash \bigwedge x_{2}$. Then (reasoning as above) $s \Vdash \bigwedge(x \cup y)$ and $x \cup y \in \mathcal{S}\left(A_{1} \wedge A_{2}\right)$. But in general, $\mathcal{S}(B \wedge C) \subseteq \mathcal{S}(B \vee C)$ (definition 3.2), and hence $x \cup y \in \mathcal{S} A$.

- The cases for $A:=\neg(A \vee B)$ and $A:=\neg(A \wedge B)$ are similar to the cases above, respectively, and the case for $A:=\neg \neg A$ is trivial.

Right-TO-LEFt. Also by induction on the complexity of $A$. Assume there's some $x \in \mathcal{S} A$ such that $s \Vdash \bigwedge x$. As before, if $A$ is a literal the result is immediate. So assume that the result holds for all $B$ less complex than $A$ and consider these cases:

- If $A:=A_{1} \wedge A_{2}$ then by definition 3.2 , for some $x_{1} \in \mathcal{S} A_{1}$ and $x_{2} \in \mathcal{S} A_{2}$, $x=x_{1} \cup x_{2}$. Given that $s \Vdash \bigwedge\left(x_{1} \cup x_{2}\right)$, we have $s \Vdash\left(\bigwedge x_{1}\right) \wedge\left(\bigwedge x_{2}\right)$ and so there are $s_{1}, s_{2}$ such that $s=s_{1} \sqcup s_{2}, s_{1} \Vdash \bigwedge x_{1}$ and $s_{2} \Vdash \bigwedge x_{2}$. By hypothesis, $s_{1} \Vdash A_{1}$ and $s_{2} \Vdash A_{2}$, and so $s \Vdash A$.

- If $A:=A_{1} \vee A_{2}$ then, by definition 3.2, either (i) $x \in \mathcal{S} A_{1}$, (ii) $x \in \mathcal{S} A_{2}$ or (iii) $x \in \mathcal{S}\left(A_{1} \wedge A_{2}\right)$. If (i) then, by hypothesis, $s \Vdash A_{1}$ and hence $s \Vdash A$. Similarly, if (ii) then $s \Vdash A_{2}$ and hence $s \Vdash A$. And if (iii) then there are 
$s_{1}, s_{2}$ such that $s=s_{1} \sqcup s_{2}, s_{1} \Vdash \bigwedge x_{1}$ and $s_{2} \Vdash \bigwedge x_{2}$. By hypothesis, $s_{1} \Vdash A_{1}$ and $s_{2} \Vdash A_{2}$, and so $s \Vdash A$.

- Again, the cases for $A:=\neg(A \vee B)$ and $A:=\neg(A \wedge B)$ are similar to the cases above, respectively, and the case for $A:=\neg \neg A$ is trivial.

§4. Characterisation of Exact Entailment. In this section, we show how to characterise exact entailment in terms of a syntactic relationship between the premises and the conclusion. The conclusion must be built from selections taken from the premises, in a certain way, in order for an exact entailment to hold. We make this relationship precise in our characterisation theorem 4.12 below. Once we've established this theorem, compactness and decidability are straightforward.

We proceed by showing how to transform a model in to a congruent model. Intuitively, the congruent model takes certain states in the original model and treats them as a single state. Our strategy is then to build a counter-model to a purported entailment by identifying all the states which truthmake the premises, and showing that this state does not truthmake the conclusion. The following definitions and lemmas give the details.

Definition 4.1 (Overlap, difference, and convexity). A state $\square$ is null just in case $\square \sqsubseteq s$ for every $s$. If a null state exists, it is unique, and we reserve the name ' $\square$ ' for it. States $s$ and $u$ overlap iff some state $t \neq \square$ is a part of both: $t \sqsubseteq s$ and $t \sqsubseteq u$. The greatest lower bound of $s$ and $u$, if there is one, is denoted $s \sqcap u$. The difference of states $s$ and $u$, written $s-u$, is the unique state $t$ (if there is one) such that any $v$ is a part of $t$ iff $v$ is a part of $s$ but does not overlap $u$. (So, $s-u$ is the $\sqsubseteq$-greatest part of $s$ which does not overlap $u$, if there is one.) A set of states $S$ is closed under difference iff $s-u \in S$ whenever $s, u \in S$. A set of states $S$ is convex iff $t \in S$ whenever $s, u \in S$ and $s \sqsubseteq t \sqsubseteq u$.

Note that $s-\square=s$ and $s-s=\square$, for any state $s$. If $S$ is closed under difference, then $s-s \in S$ for any $s \in S$, and so $\square \in S$.

For technical reasons, we will occasionally need to rely on models containing the null state. But this is a harmless move for, as the following lemma shows, we can always add the null state to the bottom of a model without changing the model elsewhere.

Lemma 4.2 (Null state). Let $M=\left\langle S, \sqsubseteq,|\cdot|^{+},|\cdot|^{-}\right\rangle$and $M^{\square}=\left\langle S \cup\{\square\}\right.$, $\complement^{\square}$ $\left.,|\cdot|^{+},|\cdot|^{-}\right\rangle$, where $s \sqsubseteq^{\square} u$ iff either $s \sqsubseteq u$ or $s=\square$. Then, for any $s \in S$ : $M, s \Vdash A$ iff $M^{\square}, s \Vdash A$.

Proof. Given lemma 3.5, we need show only that $M$ and $M^{\square}$ agree on any conjunction of literals at every $s \in S: M, s \Vdash l_{1} \wedge \cdots \wedge l_{n}$ iff $M^{\square}, s \Vdash l_{1} \wedge \cdots \wedge l_{n}$. In either model, $s \Vdash l_{1} \wedge \cdots \wedge l_{n}$ just in case there are states $s_{1}, \ldots, s_{n}$ such that $s=s_{1} \sqcup \cdots \sqcup s_{n}$ and, for each $i \leqslant n, s_{i} \Vdash l_{i}$. Given that $\square \notin V^{+} p$ and $\square \notin V^{-} p$ for any sentence letter $p, s_{i} \Vdash l_{i}$ implies $s_{i} \in S$. And since $M$ and $M^{\square}$ agree on all literals at every $s \in S$, the result follows.

Definition 4.3 (Congruence relation). Let $M=\left\langle S\right.$, ᄃ, $\left.|\cdot|^{+},|\cdot|^{-}\right\rangle$be a model with $S$ closed under arbitrary fusions $(\bigsqcup U \in S$ for any $U \subseteq S$ ) and pairwise 
difference. Let $C \subseteq S$ be a convex set closed under $\sqcup$. We define $\equiv_{C}$ to be the smallest equivalence relation on $S$ such that:

(a) If $s, u \in C$ then $s \equiv_{C} u$; and

(b) If $s \equiv_{C} s^{\prime}$ and $u \equiv_{C} u^{\prime}$ then $s \sqcup u \equiv_{C} s^{\prime} \sqcup u^{\prime}$.

(Note that there must exist a smallest such relation.) We will write $[s]_{C}$ for $\left\{u \mid u \equiv_{C} s\right\}$ and boldface $\mathbf{s}, \mathbf{u}, \ldots$ for arbitrary equivalence classes.

The following lemma gives an equivalent characterisation of $\equiv_{C}$.

Lemma 4.4. Let $M$ and $C$ be as above, and let $s \approx_{C} u$ whenever: either $s=u$; or else (i) there are $c_{1}, c_{2} \in C$ such that $c_{1} \sqsubseteq s$ and $c_{2} \sqsubseteq u$ and (ii) $s-\sqcup C=$ $u-\bigsqcup C$. Then $s \approx_{C} u$ iff $s \equiv_{C} u$.

Proof, Left-To-Right. Assume $s \approx_{C} u$. If $s=u$ then trivially, $s \equiv_{C} u$; so assume instead that $s \neq u$. Then $s-\bigsqcup C=u-\bigsqcup C$ and hence $s-\bigsqcup C \equiv_{C}$ $u-\bigsqcup C$. Moreover, there are $c_{1}, c_{2} \in C$ with $c_{1} \sqsubseteq s$ and $c_{2} \sqsubseteq u$. Hence $c_{1} \sqsubseteq$ $(s \sqcap \bigsqcup C) \sqsubseteq \bigsqcup C$. Since $C$ is $\sqcup$-closed and convex, we have $\bigsqcup C \in C$ and hence $(s \sqcap \bigsqcup C) \in C$. Similarly, we have $(u \sqcap \bigsqcup C) \in C$ and hence $(s \sqcap \bigsqcup C) \equiv_{C}$ $(u \sqcap \bigsqcup C)$. This gives us $(s-\bigsqcup C) \sqcup(s \sqcap \bigsqcup C) \equiv_{C}(u-\bigsqcup C) \sqcup(u \sqcap \bigsqcup C)$ and hence $s \equiv_{C} u$.

Right-to-left: We show $\approx_{C}$ satisfies clauses $(\mathrm{a})$ and $(\mathrm{b})$ of definition 4.3. If $s, u \in C$ then condition (i) is satisfied with $c_{1}=s$ and $c_{2}=u$ and condition (ii) is satisfied, since $s-\bigsqcup C=u-\bigsqcup C$. Hence $\approx_{C}$ satisfies clause (a). Now assume $s \approx_{C} s^{\prime}$ and $u \approx_{C} u^{\prime}$. If $s=s^{\prime}$ but $u \neq u^{\prime}$, then by definition there are $c_{1}, c_{2} \in C$ with $c_{1} \sqsubseteq u$ and $c_{2} \sqsubseteq u^{\prime}$, and $u-\bigsqcup C=u^{\prime}-\bigsqcup C$. Then $c_{1} \sqsubseteq s \sqcup u, c_{2} \sqsubseteq s^{\prime} \sqcup u^{\prime}$, and $(s \sqcup u)-\bigsqcup C=(s-\bigsqcup C) \sqcup(u-\bigsqcup C)=\left(s^{\prime}-\bigsqcup C\right) \sqcup\left(u^{\prime}-\bigsqcup C\right)=\left(s^{\prime} \sqcup u^{\prime}\right)-\bigsqcup C$, and so $s \sqcup u \approx_{C} s^{\prime} \sqcup u^{\prime}$. By similar reasoning, $s \sqcup u \approx_{C} s^{\prime} \sqcup u^{\prime}$ if $u=u^{\prime}$ but $s \neq s^{\prime}$. So now suppose $s \neq s^{\prime}$ and $u \neq u^{\prime}$. Then by definition there are $c_{1}, c_{2}, c_{3}, c_{4} \in C$ with $c_{1} \sqsubseteq s, c_{2} \sqsubseteq u, c_{3} \sqsubseteq s^{\prime}$ and $c_{4} \sqsubseteq u^{\prime}$. So $c_{1} \sqcup c_{2} \sqsubseteq s \sqcup u$ and $c_{3} \sqcup c_{4} \sqsubseteq u \sqcup u^{\prime}$ and, since $C$ is $\sqcup$-closed, we have $c_{1} \sqcup c_{2}, c_{3} \sqcup c_{4} \in C$. Moreover, from condition (ii), $s-\bigsqcup C=s^{\prime}-\bigsqcup C$ and $u-\bigsqcup C=u^{\prime}-\bigsqcup C$, hence

$$
(s-\bigsqcup C) \sqcup(u-\bigsqcup C)=\left(s^{\prime}-\bigsqcup c\right) \sqcup\left(u^{\prime}-\bigsqcup c\right)
$$

so

$$
(s \sqcup u)-\bigsqcup c=\left(s^{\prime} \sqcup u^{\prime}\right)-\bigsqcup c
$$

and hence $s \sqcup u \approx_{C} s^{\prime} \sqcup u^{\prime}$. Hence $\approx_{C}$ satisfies clause (b).

It remains only to show that $\approx_{C}$ is an equivalence relation. It is clearly reflexive and symmetrical; so suppose $s \approx_{C} t$ and $t \approx_{C} u$. If either $s=t$ or $t=u$ then $s \approx_{C} u$; so suppose this is not the case. Then by definition, we have $c_{1}, c_{2}, c_{3} \in C$ with $c_{1} \sqsubseteq s, c_{2} \sqsubseteq t$, and $c_{3} \sqsubseteq u$; and $s-\bigsqcup C=t-\bigsqcup C=u-\bigsqcup C$, hence $s \approx_{C} u$. It follows that $\approx_{C}$ is an equivalence relation and hence that $s \equiv_{C} u$ implies $s \approx_{C} u$.

Definition 4.5. Let $M$ and $C$ be as above. We define an operator $\sqcup^{C}$ on equivalence classes of states by setting $\mathbf{s} \sqcup^{C} \mathbf{u}=\bigcup_{s \in \mathbf{s}, u \in \mathbf{u}}[s \sqcup u]_{C}$.

Lemma 4.6. $[s]_{C} \sqcup^{C}[u]_{C}=[s \sqcup u]_{C}$. 
Proof. Suppose $s, s^{\prime} \in \mathbf{s}$ and $u, u^{\prime} \in \mathbf{u}$. Then $s \equiv_{C} s^{\prime}$ and $u \equiv_{C} u^{\prime}$, hence by definition, $s \sqcup u \equiv_{C} s^{\prime} \sqcup u^{\prime}$ and so $s^{\prime} \sqcup u^{\prime} \in[s \sqcup u]_{C}$. It follows that, for any $s \in \mathbf{s}$ and $u \in \mathbf{u}$ :

$$
\bigcup_{s^{\prime} \in \mathbf{s}, u^{\prime} \in \mathbf{u}}\left[s^{\prime} \sqcup u^{\prime}\right]_{C}=[s \sqcup u]_{C}
$$

and hence that $[s]_{C} \sqcup^{C}[u]_{C}=[s \sqcup u]_{C}$.

Definition 4.7 (Congruent structures). Let $M$ and $C$ be as above. We transform $M$ into a congruent structure, $M^{C}=\left\langle S^{C}, \sqsubseteq^{C},|\cdot|^{+C},|\cdot|^{-C}\right\rangle$, where:

- $S^{C}=\left\{[s]_{C} \mid s \in S\right\}$

- $\mathbf{s} \sqsubseteq^{C} \mathbf{u}$ iff $\mathbf{s} \sqcup^{C} \mathbf{u}=\mathbf{u}$

- $|p|^{+C}=\left\{\left.[s]_{C}|s \in| p\right|^{+}\right\}$

- $|p|^{-C}=\left\{\left.[s]_{C}|s \in| p\right|^{-}\right\}$

Intuitively, a congruent structure treats all the elements of $C$ as a single state, and makes whatever identifications thereby follow.

Lemma 4.8. Let $M$ and $C$ be as above. Then $M^{C}$ is a model.

Proof. Given lemma 4.6, it follows that $\sqcup^{C}$ is commutative, associative and idempotent and hence that $\complement^{C}$ is a partial order on $S^{C}$. Moreover, lemma 4.6 guarantees that $[s]_{C} \sqcup^{C}[u]_{C} \in S^{C}$ whenever $[s]_{C},[u]_{C} \in S^{C}$. It follows, by definition 2.2 , that $M^{C}$ is a model.

Lemma 4.9. Let $M^{C}$ be as above. For any state $u$ in $M^{C}$, if there is no $s \in C$ such that $s \sqsubseteq u \sqsubseteq \bigsqcup C$, then for no $s \in C$ is $s \equiv_{C} u$.

Proof. By contraposition. Suppose there is some $s \in C$ such that $s \equiv_{C} u$. Then by lemma $4.4, s \approx_{C} u$. If $s=u$ then the result follows immediately; so suppose $s \neq u$. Then by definition of $\approx_{C}$, (i) there is some $c \in C$ such that $c \sqsubseteq u$ and (ii) $s-\bigsqcup C=u-\bigsqcup C$. Since $s \in C$, we have $s \sqsubseteq \bigsqcup C$ and hence $u \sqsubseteq \bigsqcup C$, which gives us $c \sqsubseteq u \sqsubseteq \bigsqcup C$.

Lemma 4.10 (Congruent models). Let $M$ and $C$ be as in definition 4.7. We form the congruent model $M^{C}$. Then:

$$
M^{C}, \mathbf{s} \Vdash A \text { iff there is some } u \in \mathbf{s} \text { such that } M, u \Vdash A \text {. }
$$

Proof, Left-to-Right. By induction on $A$. Suppose $M^{C}, \mathbf{s} \Vdash A$. If $A:=p$ then by definition, $\mathbf{s} \in|p|^{+C}=\left\{\left.[s]_{C}|s \in| p\right|^{+}\right\}$, hence for some $u \in \mathbf{s}, u \in|p|^{+}$, and so $M, s \Vdash p$. Similarly, if $A:=\neg p$ then $\mathbf{s} \in|p|^{-C}=\left\{\left.[s]_{C}|s \in| p\right|^{-}\right\}$, hence for some $u \in \mathbf{s}, u \in|p|^{-}$, so $M, s \dashv p$, and hence $M, s \Vdash \neg p$. Now assume the result holds for all $B$ less complex than $A$.

- $A:=A_{1} \wedge A_{2}$. Then $\mathbf{s}=\mathbf{s}_{\mathbf{1}} \sqcup^{C} \mathbf{s}_{\mathbf{2}}$ where $M^{C}, \mathbf{s}_{\mathbf{1}} \Vdash A_{1}$ and $M^{C}, \mathbf{s}_{\mathbf{2}} \Vdash A_{2}$. So by hypothesis, there are $u_{1} \in \mathbf{s}_{\mathbf{1}}$ and $u_{2} \in \mathbf{s}_{\mathbf{2}}$ such that $M, u_{1} \Vdash A_{1}$ and $M, u_{2} \Vdash A_{2}$. Hence $M, u_{1} \sqcup u_{2} \Vdash A$. Then we have $\mathbf{s}=\mathbf{s}_{\mathbf{1}} \sqcup^{C} \mathbf{s}_{\mathbf{2}}=$ $\left[u_{1}\right]_{C} \sqcup^{C}\left[u_{2}\right]_{C}=\left[u_{1} \sqcup u_{2}\right]^{C}$ (by lemma 4.6), and hence $u_{1} \sqcup u_{2} \in \mathbf{s}$.

- $A:=A_{1} \vee A_{2}$. Then (i) $M^{C}$, $\mathbf{s} \Vdash A_{1}$, (i) $M^{C}$, $\mathbf{s} \Vdash A_{2}$, or (iii) $\mathbf{s}=\mathbf{s}_{\mathbf{1}} \sqcup^{C} \mathbf{s}_{\mathbf{2}}$ where $M^{C}, \mathbf{s}_{\mathbf{1}} \Vdash A_{1}$ and $M^{C}, \mathbf{s}_{\mathbf{2}} \Vdash A_{2}$. If (i) then, by hypothesis, $M, u \Vdash$ $A_{1}$, and hence $M, u \Vdash A$, for some $u \in \mathbf{s}$. Similarly, if (ii) then $M, u \Vdash A_{2}$, and hence $M, u \Vdash A$, for some $u \in \mathbf{s}$. If (iii) then, by the reasoning in the 
previous case, we have $u_{1} \sqcup u_{2} \in \mathbf{s}$ such that $M, u_{1} \Vdash A_{1}$ and $M, u_{2} \Vdash A_{2}$, and hence $M, u_{1} \sqcup u_{2} \Vdash A$.

- The cases for $A:=\neg(A \vee B)$ and $A:=\neg(A \wedge B)$ are similar to the cases above, respectively, and the case for $A:=\neg \neg A$ is trivial.

Right-to-left. Again, by induction on $A$. Assume $M, u \Vdash A$ for some $u \in \mathbf{s}$. Then $\mathbf{s}=[u]_{C}$. If $A:=p$ then by definition, $u \in|p|^{+}$, so $[u]_{C} \in|p|^{+C}$, so $\mathbf{s} \in|p|^{+C}$, and hence $M^{C}, \mathbf{s} \Vdash p$. Similarly, if $A:=\neg p$ then $u \in|p|^{-}$, so $\mathbf{s} \in|p|^{-C}$, hence $M^{C}, \mathbf{s} \dashv p$ and so $M^{C}, \mathbf{s} \Vdash \neg p$. Now assume the result holds for all $B$ less complex than $A$.

- $A:=A_{1} \wedge A_{2}$. Then $u=u_{1} \sqcup u_{2}$, where $M, u_{1} \Vdash A_{1}$ and $M, u_{2} \Vdash A_{2}$. By hypothesis, $M^{C},\left[u_{1}\right]_{C} \Vdash A_{1}$ and $M^{C},\left[u_{2}\right]_{C} \Vdash A_{2}$, and hence $M^{C},\left[u_{1}\right]_{C} \sqcup^{C}$ $\left[u_{2}\right]_{C} \Vdash A$. By lemma 4.6, $\left[u_{1}\right]_{C} \sqcup^{C}\left[u_{2}\right]_{C}=\left[u_{1} \sqcup u_{2}\right]_{C}=[u]_{C}=\mathbf{s}$, hence $M^{C}, \mathbf{s} \Vdash A$.

- $A:=A_{1} \vee A_{2}$. Then (i) $M, u \Vdash A_{1}$, (ii) $M, u \Vdash A_{2}$, or (iii) $u=u_{1} \sqcup u_{2}$ where $M, u_{1} \Vdash A_{1}$ and $M, u_{2} \Vdash A_{2}$. If (i) then, by hypothesis, $M^{C}, \mathbf{s} \Vdash A_{1}$ and hence $M^{C}, \mathbf{s} \Vdash A$. Similarly, if (ii) then $M^{C}, \mathbf{s} \Vdash A_{2}$ and hence $M^{C}, \mathbf{s} \Vdash$ $A$. If (iii) then, by reasoning in the previous case, $M^{C},\left[u_{1}\right]_{C} \Vdash A_{1}$ and $M^{C},\left[u_{2}\right]_{C} \Vdash A_{2}$, hence $M^{C},\left[u_{1}\right]_{C} \sqcup^{C}\left[u_{2}\right]_{C} \Vdash A$, and $\left[u_{1}\right]_{C} \sqcup^{C}\left[u_{2}\right]_{C}=\mathbf{s}$. So $M^{C}, \mathbf{s} \Vdash A$.

- Again, the cases for $A:=\neg(A \vee B)$ and $A:=\neg(A \wedge B)$ are similar to the cases above, respectively, and the case for $A:=\neg \neg A$ is trivial.

We now form a congruence relation on our canonical model, transforming the latter into a congruent canonical model. Note that, since states in the canonical model are sets of sentences, the set of all such states is trivially closed under arbitrary fusions and difference; and we can always take a set of canonical model states $S$ and close it under convexity and pairwise fusion. We use the resulting set of states, $C$, to form our convex canonical model.

Corollary 4.11. Let $\mathfrak{M}=\left\langle\mathfrak{S}, \subseteq,|\cdot|^{+},|\cdot|^{-}\right\rangle$be the canonical model, and $C \subseteq \mathfrak{S}$ be convex and closed under $\cup$. Form the congruent canonical model $\mathfrak{M}^{C}$. Then $\mathfrak{M}^{C}, \mathbf{s} \Vdash A$ iff $\mathbf{s} \cap \mathcal{S} A \neq \varnothing$.

Proof. From lemma 4.10 and lemma 3.5 , we have $\mathfrak{M}^{C}, \mathbf{s} \Vdash A$ iff $\mathfrak{M}, u \Vdash A$ for some $u \in \mathbf{s}$ iff $\mathfrak{M}, u \Vdash \bigwedge x$ for some $u \in \mathbf{s}$ and $x \in \mathcal{S} A$. But by construction of $\mathfrak{M}$, this holds iff $x=u$ for some $u \in \mathbf{s}$ and $x \in \mathcal{S} A$ and hence iff $\mathbf{s} \cap \mathcal{S} A \neq \varnothing . \quad \dashv$

Now for our characterisation theorem, which says the following. Suppose we have an entailment from premises $\Gamma$ to conclusion $A$. Then, for any selection $X$ from the premises, we must be able to find a selection $y$ from the conclusion which lies between some specific $x \in X$ and $X$ itself: $x \subseteq y \subseteq \bigcup X$. Intuitively, we may think of selections as describing specific truth-grounds for a sentence. So our theorem intuitively says: an entailment holds when, for any collection of truthgrounds for the premises, some truth-ground for the conclusion lies in-between one and all of those premise truth-grounds.

Let us illustrate the idea. Consider the entailment: $p, r \models(p \vee q) \wedge r$. The selections for $p$ and $r$ are just $\{p\}$ and $\{r\}$ (neither of which is a selection from 
$(p \vee q) \wedge r)$. But $\{\{p\},\{r\}\}$ is the only selection from the premise-set $\{p, r\}$ and $\{p, r\}$ is a selection from the conclusion. This gives us the inclusion relationship $\{p\} \subseteq\{p, r\} \subseteq \bigcup\{\{p\},\{r\}\}$, which satisfies the theorem's condition on entailment, hence $p, r \models(p \vee q) \wedge r$.

Theorem 4.12 (Characterisation of $\models$ ). $\Gamma \models A$ if and only if, for any $X \in$ $\mathcal{S} \Gamma$, some $y \in \mathcal{S} A$ and some $x \in X: x \subseteq y \subseteq \bigcup X$.

Proof, Left-to-Right. We show the contrapositive. Assume that, for some $X \in \mathcal{S} \Gamma$ (which we keep fixed) and any $y \in \mathcal{S} A$, there is no $x \in X$ such that $x \subseteq y \subseteq \bigcup X$. Now let $C$ be the closure of $X$ under $\sqcup,-$, and convexity. Then $\bigcup C=\bigcup X$ and, for any $z \in C$, there is some $x \in X$ with $x \subseteq z$ and so, for any $y \in \mathcal{S} A$, there is no $x \in C$ such that $x \subseteq y \subseteq \bigcup C$. Now let $\mathfrak{M}^{C}$ be the congruent canonical model over $C$. Then $C$ is a state of $\mathfrak{M}^{C}, C=[x]_{C}$ for any $x \in C$, and there is no $x \in C$ and $y \in \mathcal{S} A$ such that $x \sqsubseteq y \sqsubseteq \bigsqcup C$. So, by lemma 4.9, for no $x \in C$ and $y \in \mathcal{S} A$ do we have $x \equiv_{C} y$. It follows that $\mathcal{S} A \cap C=\varnothing$ and so, by corollary 4.11, $\mathfrak{M}^{C}, C \Downarrow A$. Moreover, for each $B \in \Gamma$, we have $\mathcal{S} B \cap X \neq \varnothing$, hence $\mathcal{S} B \cap C \neq \varnothing$ and so, by corollary 4.11, $\mathfrak{M}^{C}, C \Vdash B$. It follows that $\Gamma \not \models A$.

Right-TO-LEFT. Assume the right-hand-side of the theorem and take any model $M$ and state $s$ in $M$ and suppose that $s \Vdash \Gamma$. We show $s \Vdash A$. For each $B \in \Gamma$, we have $s \Vdash B$ and so, by lemma 3.5, $s \Vdash \bigwedge x$ for some $x \in \mathcal{S} B$. So for some $X \in \mathcal{S} \Gamma$, we have $s \Vdash \bigwedge x$ for each $x \in X$ : we now hold this $X$ fixed. By assumption, for some $y \in \mathcal{S} A$ and some $x \in X$, we have $x \subseteq y \subseteq \bigcup X$. Moreover, $y-x \subseteq z$ for some $z \in X$ and hence $s \Vdash \bigwedge z$. So for any $l \in y-x$, there is some $t \sqsubseteq s$ such that $t \Vdash l$. It follows that there is some $u \sqsubseteq s$ such that $u \Vdash \wedge(y-x)$, hence $s \sqcup u \Vdash \wedge x \wedge \bigwedge(y-x)$ and so $s \Vdash \bigwedge y$. Since $y \in \mathcal{S} A$, lemma 3.5 gives us $s \Vdash A$. It follows that $\Gamma \models A$.

$\S 5$. Compactness and Decidability. We turn to compactness. Note that we cannot meaningfully express compactness in terms of satisfiability (i.e., by saying that any set of formulas is satisfiable iff all its finite subsets are) since, as we have already noted, any set of formulas is satisfiable in the truthmaking semantics. So we present our compactness result (theorem 5.2 below) in terms of entailment.

Lemma 5.1. Let $T$ be a constant verified and falsified by every state in every model. For any formula $A$ and set of sentence letters $P$, let $A[T / P]$ be the formula obtained from $A$ by uniformly substituting $\top$ for each $p \in P$ in $A$. Then $A \models$ $A[\top / P]$.

Proof. By induction on $A$. The base case holds trivially, so suppose the result holds for all $B$ less complex than $A$, suppose $s \Vdash A$, and consider these cases.

- $A:=A_{1} \wedge A_{2}$. Then $s=s_{1} \sqcup s_{2}$ where $s_{1} \Vdash A_{1}$ and $s_{2} \Vdash A_{2}$. By hypothesis, $s_{1} \Vdash A_{1}\left[{ }^{\top} / P\right]$ and $s_{2} \Vdash A_{2}\left[{ }^{\top} / P\right]$, hence $s \Vdash A_{1}\left[{ }^{\top} / P\right] \wedge A_{2}\left[{ }^{\top} / P\right]$ and so $s \Vdash A[\top / P]$.

- $A:=A_{1} \vee A_{2}$. Then $s \Vdash A_{1}, s \Vdash A_{2}$, or else $s=s_{1} \sqcup s_{2}$ where $s_{1} \Vdash A_{1}$ and $s_{2} \Vdash A_{2}$. If $s \Vdash A_{1}$ then by hypothesis $s \Vdash A_{2}\left[{ }^{\top} / P\right]$, hence $s \Vdash A_{1}[\mathrm{~T} / P] \vee$ $A_{2}[\mathrm{~T} / P]$ and so $s \Vdash\left(A_{1} \vee A_{2}\right)[\top / P]$. Similarly if $s \Vdash A_{2}$. Otherwise, by 
hypothesis $s_{1} \Vdash A_{1}[\mathrm{~T} / P]$ and $s_{2} \Vdash A_{2}[\mathrm{~T} / P]$, hence $s \Vdash A_{1}[\mathrm{~T} / P] \vee A_{2}[\mathrm{~T} / P]$ and so $s \Vdash A[\top / P]$.

- Again, the cases for $A:=\neg(A \vee B)$ and $A:=\neg(A \wedge B)$ are similar to the cases above, respectively, and the case for $A:=\neg \neg A$ is trivial.

Theorem 5.2 (Compactness). If $\Gamma \models A$ then $\Delta \models A$ for some finite $\Delta \subseteq \Gamma$.

Proof. Fix the conclusion $A$, and for any formula $B$, let $B^{A}$ be the result of substituting $\top$ in $B$ for any sentence letter not in $A$. Let $\Gamma^{A}=\left\{B^{A} \mid B \in \Gamma\right\}$. Since $\models$ is closed under uniform substitution, $\Gamma^{A} \models A$. We then partition $\Gamma^{A}$ into equivalence classes and pick one sentence from each class: let the resulting set be $\Delta^{A}$. Then $\Delta^{A} \models A$ too. And since $\Gamma^{A}$ is constructed from a finite set of sentence letters, there are finitely many such equivalence classes and so $\Delta^{A}$ is finite. Now, for each $B^{A} \in \Delta^{A}$, we pick a corresponding $B^{\prime} \in \Gamma$, such that $B^{\prime A}=B^{A}$. (Note that $B^{A}$ need not uniquely determine this $B^{\prime}$.) Let $\Delta$ be the set of the chosen $B^{\prime}$ s. Then $\Delta \subseteq \Gamma$ is finite. Now suppose $s \Vdash \Delta$. Then, by lemma 5.1, $s \Vdash \Delta^{A}$, hence $s \Vdash \Gamma^{A}$ and so $s \Vdash A$. It follows that $\Delta \models A$.

Theorem 5.3 (Decidability). For finite $\Gamma$, it is decidable whether $\Gamma \models A$.

Proof. By theorem 4.12, there is a correct algorithm for checking whether $\Gamma \vDash A$ which cycles through each $X \in \mathcal{S} \Gamma$, checking whether there is some $x \in X$ and some $y \in \mathcal{S} A$ such that $x \subseteq y \subseteq \bigcup X$. Each such $x, y$ and $\bigcup X$ is a finite set, so each such check is a finite procedure. Since there are at most $|\mathcal{S} \Gamma| \cdot \max \{|X| \mid X \in \mathcal{S} \Gamma\} \cdot|\mathcal{S} A|$ such checks to perform, the procedure will terminate after finitely many steps.

§6. Non-Vacuous and Convex Models. In this section, we consider two conditions in turn, non-vacuity and convexity, and investigate whether they affect the exact entailment relation.

Non-Vacuous Models. In non-vacuous models, every sentence letter $p$ has a truthmaker and a falsemaker:

Definition 6.1 (Non-vacuous models). A model $M=\left\langle S, \sqsubseteq,|\cdot|^{+},|\cdot|^{-}\right\rangle$is non-vacuous iff, for each sentence letter $p$ in the language, both $|p|^{+}$and $|p|^{-}$ are nonempty.

In a non-vacuous model $M$, the sets $|A|^{+}$and $|A|^{-}$are non-empty for arbitrary formulas $A$ (as the reader can easily check).

Definition 6.2 (Non-vacuous exact entailment).

We show that non-vacuous exact entailment is exact entailment:

Theorem 6.3. $\Gamma \models_{n v} A$ if and only if $\Gamma \models A$.

Proof, Left-TO-RIght. By contraposition. Suppose $\Gamma \not \models A$. Then there is a model $M$ and state $s$ in $M$ such that $M, s \Vdash \Gamma$ but $M, s \Downarrow \forall$. We transform $M$ into $M^{*}$ by adding a single state, $t$, such that $u \sqsubset t$ for each state $u$ in $M$, and adding $t$ to both $|p|^{+}$and $|p|^{-}$for every sentence letter $p$. Then $t \sqcup u=t$ for each state $u$ in $M^{*}$, hence each pair of states in $M^{*}$ has a least upper bound. This 
guarantees that $M^{*}$ is a genuine model. Moreover, $M^{*}$ is non-vacuous, since $t \in|p|^{+} \cap|p|^{-}$for each $p$. Now we show, by induction on $A$, that $M^{*}, u \Vdash A$ iff $M, u \Vdash A$ for each $u$ in $M$.

In the only if direction, the base case holds, given that $t$ is not in $M$. So suppose the result holds for all $B$ less complex than $A$, and consider these cases.

- $A:=A_{1} \wedge A_{2}$. Then $u=u_{1} \sqcup u_{2}$ where $M^{*}, u_{1} \Vdash A_{1}$ and $M^{*}, u_{2} \Vdash A_{2}$. So by hypothesis, $M, u_{1} \Vdash A_{1}$ and $M, u_{2} \Vdash A_{2}$, hence $M, u \Vdash A$.

- $A:=A_{1} \vee A_{2}$. Then $M^{*}, s \Vdash A_{1}, M^{*}, s \Vdash A_{2}$, or $u=u_{1} \sqcup u_{2}$ where $M^{*}, u_{1} \Vdash A_{1}$ and $M^{*}, u_{2} \Vdash A_{2}$. So by hypothesis, $M, u \Vdash A_{1}, M, u \Vdash A_{2}$, or else $M, u_{1} \Vdash A_{1}$ and $M, u_{2} \Vdash A_{2}$. Either way, we have $M, u \Vdash A$.

- The $A:=\neg\left(A_{1} \vee A_{2}\right)$ and $A:=\neg\left(A_{1} \wedge A_{2}\right)$ cases are similar, and the $A:=\neg \neg A_{1}$ case is trivial.

The if direction is similar. So, given that $M, s \Vdash \Gamma$ but $M, s \Downarrow A$, we have $M^{*}, s \Vdash \Gamma$ and $M^{*}, s \Downarrow \forall$, and hence $\Gamma \not \models_{n v} A$.

Right-to-left: Trivial, given that all non-vacuous models are models.

It is worth noting that the proof of theorem 4.12 gives us an alternative proof of theorem 6.3, given that the congruent canonical model used in that proof is non-vacuous.

Convex Models. Our current understanding of truthmaking allows structures like the following, where the shaded area marks truthmakers for $(p \wedge q) \vee r$ :

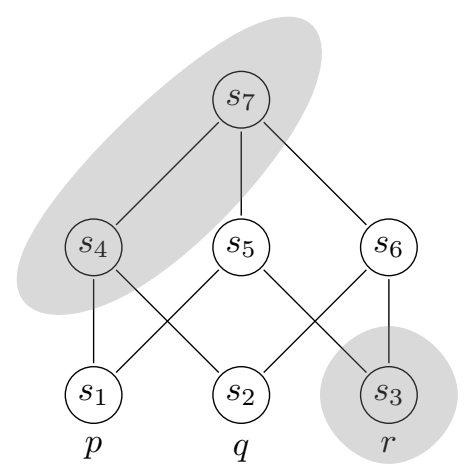

Here, $s_{7}$ has parts $\left(s_{5}\right.$ and $\left.s_{6}\right)$ which do not truthmake $(p \wedge q) \vee r$, each of which has a further part $\left(s_{3}\right)$ which does truthmake $(p \wedge q) \vee r$. So, we can begin with a truthmaker for $(p \wedge q) \vee r$, add to it so that it will no longer truthmake $(p \wedge q) \vee r$, and add to it further, so that it will again truthmake $(p \wedge q) \vee r$. In other words, the set of states $|(p \wedge q) \vee r|^{+}$is not convex.

We can work instead with the convex closure of the truthmaking and falsemaking sets (definition 4.1), which we denote $|A|_{*}^{+}$and $|A|_{*}^{-}$. These obey a selection lemma:

Lemma 6.4 (Convex selection lemma). For any formula $A$, model $M$ and state $s$ in $M: s \in|A|_{*}^{+}$iff there are $x, y \in \mathcal{S} A$ \& states $t \sqsubseteq s \sqsubseteq u$ such that $t \Vdash \bigwedge x \mathcal{E}$ $u \Vdash \wedge y$. 
Proof, Left-TO-Right. Assume $s \in|A|_{*}^{+}$. Then by definition there are $t, u \in$ $|A|^{+}$with $t \sqsubseteq s \sqsubseteq u$. Since $t \Vdash A$ and $u \Vdash A$, by lemma 3.5 there are $x, y \in \mathcal{S} A$ such that $t \Vdash \bigwedge x$ and $u \Vdash \bigwedge y$.

Right-TO-LEFT. Assume there are $x, y \in \mathcal{S} A$ and states $t \sqsubseteq s \sqsubseteq u$ such that $t \Vdash \bigwedge x$ and $u \Vdash \bigwedge y$. By lemma 3.5, $t \Vdash A$ and $u \Vdash A$ and so, by definition, $s \in|A|_{*}^{+}$.

We use these notions to define convex exact entailment, and show that it coincides with exact entailment:

Definition 6.5 (Convex exact entailment). $\Gamma$ convex exactly entails $A, \Gamma \models_{*}$ $A$, iff for any model $M:|A|_{*}^{+} \subseteq|\Gamma|_{*}^{+}$in $M$.

Theorem 6.6 (Convex exact entailment). $\Gamma \models_{*} A$ iff $\Gamma \models A$.

Proof, Left-to-Right. Suppose $\Gamma \not \models A$. Then there is a model $M=\langle S$, $\left.,|\cdot|^{+},|\cdot|^{-}\right\rangle$and state $s$ in $M$ such that $M, s \Vdash \Gamma$ but $M, s \| A$. Let $M^{s}$ be the model just like $M$ except restricted to states $u \sqsubseteq s$. Then, for all $u \sqsubseteq s$ in $M$, $M^{s}, u \Vdash A$ iff $M, u \Vdash A$. Hence $M^{s}, s \Vdash \Gamma$ but $M^{s}, s \Downarrow A$, so $s \notin|A|^{+}$and so (since $s$ is the top state in $\left.M^{s}\right) s \notin|A|_{*}^{+}$. Since $|\Gamma|^{+} \subseteq|\Gamma|_{*}^{+}, \Gamma \not{ }_{*} A$.

Right-to-left. Suppose $\Gamma \models A$ and $s \in|\Gamma|_{*}^{+}$. Then, for each $B \in \Gamma$, there are states $s_{1}^{B} \sqsubseteq s \sqsubseteq s_{2}^{B}$ such that $s_{1}^{B} \Vdash B$ and $s_{2}^{B} \Vdash B$. By lemma 3.5, for each $B \in \Gamma$, there are selections $x_{1}^{B}, x_{2}^{B} \in \mathcal{S} B$ such that $s_{1}^{B} \Vdash \bigwedge x_{1}^{B}$ and $s_{2}^{B} \Vdash \bigwedge x_{2}^{B}$. Let $X_{1}=\left\{x_{1}^{B} \mid B \in \Gamma\right\}$ and $X_{2}=\left\{x_{2}^{B} \mid B \in \Gamma\right\}$. Then $X_{1}, X_{2} \in \mathcal{S} \Gamma$. So by theorem 4.12, there are $y_{1}, y_{2} \in \mathcal{S} A, z_{1} \in X_{1}$, and $z_{2} \in X_{2}$ such that $z_{1} \subseteq y_{1} \subseteq \bigcup X_{1}$ and $z_{2} \subseteq y_{2} \subseteq \bigcup X_{2}$. For some $B^{\prime}, B^{\prime \prime} \in \Gamma, z_{1}=x_{1}^{B^{\prime}}$ and $z_{2}=x_{2}^{B^{\prime \prime}}$ and hence $s_{1}^{B^{\prime}} \Vdash \bigwedge z_{1}$ and $s_{2}^{B^{\prime \prime}} \Vdash \bigwedge z_{2}$.

Now take any literal $l \in \bigcup X_{1}$. Then $l \in x_{i}^{B}$ for some $B \in \Gamma$ and $x_{i} \in \mathcal{S} B$. So there is a state $s^{B} \sqsubseteq s$ such that $s^{B} \Vdash \bigwedge x_{i}^{B}$ and, since $x_{i}^{B}$ is finite, there is a state $s^{\prime} \sqsubseteq s^{B} \sqsubseteq s$ such that $s^{\prime} \Vdash l$. Now consider $y_{1}-z_{1}$. This is a finite set of literals $\left\{l_{1}, \ldots, l_{n}\right\} \subseteq X_{1}$ for which, by the preceding reasoning, there are states $t_{i} \sqsubseteq s$ such that $t_{i} \Vdash l_{i}$ for each $i \leqslant n$, and hence there is a state $t=\left(t_{1} \sqcup \cdots \sqcup t_{n}\right) \sqsubseteq s$ such that $t \Vdash \bigwedge\left(y_{1}-z_{1}\right)$. Similarly, there is some $u \sqsubseteq s$ such that $u \Vdash \bigwedge\left(y_{2}-z_{2}\right)$. Then $s_{1}^{B^{\prime}} \sqcup t \Vdash \bigwedge y_{1}$ and $s_{2}^{B^{\prime \prime}} \sqcup u \Vdash \bigwedge y_{2}$. But $s_{1}^{B^{\prime}} \sqsubseteq s \sqsubseteq s_{2}^{B^{\prime \prime}}$ and so $\left(s_{1}^{B^{\prime}} \sqcup t\right) \sqsubseteq s \sqsubseteq\left(s_{2}^{B^{\prime \prime}} \sqcup u\right)$. By lemma 3.5, $s_{1}^{B^{\prime}} \sqcup t \Vdash A$ and $s_{2}^{B^{\prime \prime}} \sqcup u \Vdash A$, and so $s \in|A|_{*}^{+}$. Hence $\Gamma \models_{*} A$.

An Alternative Approach to Convexity. In defining $\models_{*}$, we have not changed our truthmaking or falsemaking clauses. There is an alternative approach, in which we replace these with truthmaking and falsemaking relations $\Vdash_{c v x}$ and $\dashv_{c v x}$, defined recursively:

DEFINITION 6.7.

$s \Vdash_{c v x} A \vee B$ iff either $s \Vdash_{c v x} A$; or $s \Vdash_{c v x} B$; or there are $t$, u such that:

$t \Vdash_{c v x} A, u \Vdash_{c v x} B$ and either $t \sqsubseteq s \sqsubseteq t \sqcup u$ or $u \sqsubseteq s \sqsubseteq t \sqcup u$

$s \dashv_{c v x} A \wedge B$ iff either $s \dashv_{c v x} A$; or $s \dashv_{c v x} B$; or there are $t, u$ such that:

$t \dashv_{c v x} A, u \dashv_{c v x} B$ and either $t \sqsubseteq s \sqsubseteq t \sqcup u$ or $u \sqsubseteq s \sqsubseteq t \sqcup u$

The remaining $\Vdash_{c v x}$ and $\dashv_{c v x}$ clauses are identical to the $\Vdash$ and $\dashv$ clauses given in definition $2.3, \S 2$. 
In general, these are not convex relations. In the model below, $s_{1} \Vdash_{c v x} p \wedge q$, $s_{5} \Vdash_{c v x} p \wedge q, s_{1} \sqsubseteq s_{3} \sqsubseteq s_{5}$, and yet $s_{3} \nvdash_{c v x} p \wedge q$ :

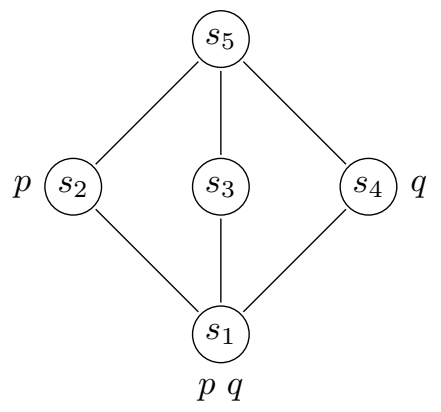

This model is non-distributive, for $s_{3} \sqcap\left(s_{2} \sqcup s_{4}\right)=s_{3} \neq s_{1}=\left(s_{3} \sqcap s_{2}\right) \sqcup\left(s_{3} \sqcap\right.$ $s_{4}$ ). And in general, we can show that any counter-model to convexity is nondistributive. Restricting to distributive models, the truthmaking and falsemaking sets defined over $\Vdash_{c v x}$ and $\dashv_{c v x}$ are convex, and provide us with an alternative definition of convex exact entailment.

Definition 6.8 (Distributive models). A model $M=\left\langle S, \sqsubseteq,|\cdot|^{+},|\cdot|^{-}\right\rangle$is distributive iff, for all $s, t, u \in S: s \sqcap(t \sqcup u)=(s \sqcap t) \sqcup(s \sqcap u)$.

Definition 6.9. $\Gamma \models_{c v x} A$ iff for every distributive model $M$ in which $|p|^{+}$ and $|p|^{-}$are convex for every sentence letter $p$, and for every state $s$ in $M$ : $M, s \Vdash c v x$ A only if $M, s \Vdash c v x \Gamma$.

Lemma 6.10 (Convexity). In a distributive model $M$ for which $|p|^{+}$and $|p|^{-}$ are convex for every sentence letter $p,\left\{s \mid s \Vdash_{c v x} A\right\}=|A|_{*}^{+}$and $\left\{s \mid s \dashv_{c v x}\right.$ $A\}=|A|_{*}^{-}$for every $A$.

Proof. The proof, which we omit here, shows that $\left\{s \mid s \Vdash_{c v x} A\right\} \subseteq|A|_{*}^{+}$ and that $|A|_{*}^{+} \subseteq\left\{s \mid s \Vdash_{c v x} A\right\}$ by induction on $A$. We note that the only case to make use of distribution is the conjunction case for $|A|_{*}^{+} \subseteq\left\{s \mid s \Vdash_{c v x} A\right\} . \quad-1$

Corollary 6.11. In a distributive model in which $|p|^{+}$and $|p|^{-}$are convex for every sentence letter $p$, if $s \Vdash_{c v x} A, u \Vdash_{c v x} A$, and $s \sqsubseteq t \sqsubseteq u$, then $t \Vdash_{c v x} A$.

Proof. Immediate from lemma 6.10.

Theorem 6.12. $\Gamma \models_{c v x} A$ iff $\Gamma \models_{*} A$ iff $\Gamma \models A$.

Proof. Immediate from lemma 6.10 and theorem 6.6.

§7. Non-Vacuous Convex Models. We have seen that both non-vacuous exact entailment and convex exact entailment coincide with exact entailment. But when we combine both conditions, non-vacuity and convexity, we obtain a different consequence relation, which we investigate in this section.

Definition 7.1 (Non-vacuous convex exact entailment). $\Gamma$ non-vacuously convex entails $A, \Gamma \models_{n v *} A$, just in case, for every non-vacuous model $M:|A|_{*}^{+} \subseteq$ $|\Gamma|_{*}^{+}$in $M$. 
We showed above that $\models$ coincides with both $\models_{n v}$ and $\models_{*}$. However, it does not coincide with $\models_{n v *}$. Non-vacuous convex entailment supports inferences

$$
\bigwedge x \models_{n v *} \bigwedge x_{1} \vee \bigwedge x_{2}
$$

for any sets of literals $x, x_{1}, x_{2}$ such that $x_{1} \subseteq x \subseteq x_{2}$ (whereas exact entailment need not support such inferences). For example, we have

$$
p \vee(p \wedge q) \vee(p \wedge q \wedge r) \models_{n v *} p \vee(p \wedge q \wedge r)
$$

and yet, as the reader can easily check,

$$
p \vee(p \wedge q) \vee(p \wedge q \wedge r) \not p p \vee(p \wedge q \wedge r)
$$

We now prepare for a syntactic characterisation of non-vacuous convex entailment, along the lines of theorem 4.12 .

Lemma 7.2 (Congruent canonical model convexity). For any set of sets of literals $C \subseteq 2^{\Lambda}$, let $\mathfrak{M}^{C}$ be the congruent canonical model over $C$. Then, for any sets of literals $x, y$ and states $\mathbf{s} \neq C$, $\mathbf{t}$, and $\mathbf{u}$ in $\mathfrak{M}^{C}:$ if $\mathbf{s} \sqsubseteq \mathbf{t} \sqsubseteq \mathbf{u}, \mathbf{s} \Vdash \wedge x$, and $\mathbf{u} \Vdash \wedge z$, then there is some $y$ such that $x \subseteq y \subseteq z$ and $\mathbf{t} \Vdash \wedge y$.

Proof. Assume $\mathbf{s} \sqsubseteq \mathbf{t} \sqsubseteq \mathbf{u}, \mathbf{s} \Vdash \bigwedge x$, and $\mathbf{u} \Vdash \bigwedge z$ in $\mathfrak{M}^{C}$. Then by construction of $\mathfrak{M}^{C}, \mathbf{s}=[x]_{C}$ and $\mathbf{u}=[z]_{C}$ and, for some $y, \mathbf{t}=[y]_{C}$. By definition, $[z]_{C}=[y]_{C} \sqcup^{C}[z]_{C}=[y \cup z]_{C}$, so $z \equiv_{C} y \cup z$, so by lemma 4.4, $z \approx_{C} y \cup z$. Then by definition, either (i) $z=y \cup z$ (so $y \subseteq z$ ) or else (ii) there are $c_{1}, c_{2} \in C$ such that $c_{1} \subseteq z, c_{2} \subseteq(y \cup z)$, and $z-\bigcup C=(y \cup z)-\bigcup C$. So, if $y \nsubseteq z$, then $y \in C$ and $z \cap \bigcup C \neq \varnothing$. Either way, there is some $y^{\prime} \in[y]_{C}$ such that $y^{\prime} \subseteq z$. Since $\left[y^{\prime}\right]_{C}=[y]_{C}$, we have $[x]_{C} \sqsubseteq\left[y^{\prime}\right]_{C}$. By the same reasoning, if $x \nsubseteq y$, then $x \in C$ and $y \cap \bigcup C \neq \varnothing$. But by assumption, $[x]_{C} \neq C$, hence $x \notin C$, hence $x \subseteq y^{\prime} \subseteq z$. Finally, by lemma $4.10,[y]_{C} \Vdash \wedge y^{\prime}$ and hence $\mathbf{t} \Vdash \wedge y^{\prime}$.

Our previous characterisation theorem (4.12) for $\models$ fails for $\models_{n v *}$, for it requires (in the case of single-premise inferences, $A \models B$ ) that every selection from $A$ to be a selection from $B$ too. But non-vacuous convex exact entailment relaxes this requirement: each selection from the premise must be in-between (wrt $\subseteq$ ) selections from the conclusion. This suggests that we should characterise $\Gamma \models_{n v *} A$ in terms of the convex closure of $\mathcal{S} A, \mathcal{S}_{*} A$, in place of $\mathcal{S} A$. Theorem 7.4 below generalises this idea to the multiple-premise case. (Note that 4.12 is a special case of 7.4 , for which $y \in \mathcal{S} A$.)

Definition 7.3. For any $A, \mathcal{S}_{*} A$ is the convex closure (wrt $\subseteq$ ) of $\mathcal{S} A$ : the smallest set of sets of literals such that $y \in \mathcal{S}_{*} A$ whenever there are $x, z \in \mathcal{S}_{*} A$ such that $x \subseteq y \subseteq z$.

Theorem 7.4 (Characterisation of $\models_{n v *}$ ). $\Gamma \models_{n v *} A$ if and only if, for any $X \in \mathcal{S} \Gamma$, some $x \in X$ and some $y \in \mathcal{S}_{*} A: x \subseteq y \subseteq \bigcup X$.

Proof, Left-to-Right. Assume that, for some $X \in \mathcal{S}_{*} \Gamma$ (which we keep fixed) and any $y \in \mathcal{S}_{*} A$, there is no $x \in X$ such that $x \subseteq y \subseteq \bigcup X$. Let $C$ be the $\sqcup,-$, and convex closure of $X$, and $\mathfrak{M}^{C}$ be the congruent canonical model over $C$. Following the proof of theorem 4.12, for any $y \in \mathcal{S}_{*} A$, there is no $s \in C$ such that $y \equiv_{C} s$, hence $\mathfrak{M}^{C}, C \Downarrow \wedge y$. Now suppose, for reductio, that $C \in|A|_{*}^{+}$. Then, by lemma 6.4, there are selections $y_{1}, y_{2} \in \mathcal{S} A$ and states $\mathbf{s} \sqsubseteq C \sqsubseteq \mathbf{u}$ in $\mathfrak{M}^{C}$ 
such that $\mathbf{s} \in\left|\bigwedge y_{1}\right|_{*}^{+}$and $\mathbf{u} \in\left|\bigwedge y_{2}\right|_{*}^{+}$. Then $\mathfrak{M}^{C}, \mathbf{s} \Vdash \bigwedge y_{1}$ and $\mathfrak{M}^{C}, \mathbf{u} \Vdash \bigwedge y_{2}$. Given the reasoning above, $\mathbf{s} \neq C \neq \mathbf{u}$ and so, by lemma 7.2, there is a $y$ such that $y_{1} \subseteq y \subseteq y_{2}$ and $\mathfrak{M}^{C}, C \Vdash \bigwedge y$. But, since $y_{1}, y_{1} \in \mathcal{S} A$, it follows that $y \in \mathcal{S}_{*} A$ : contradiction. So $C \notin|A|_{*}^{+}$. Moreover (by the proof of theorem 4.12), $C \in|\Gamma|^{+}$, hence $C \in|\Gamma|_{*}^{+}$. Since $\mathfrak{M}^{C}$ is non-vacuous, we have $\Gamma \not \#_{n v *} A$.

Right-TO-LEFT. Assume the right-hand-side of the theorem and take any non-vacuous model $M$ and state $s$ such that $s \in|\Gamma|_{*}^{+}$. By lemma 6.4, for each $B \in \Gamma$, there are $x, y \in \mathcal{S} A$ and states $t \sqsubseteq s$ and $u \sqsupseteq s$ such that $t \Vdash \bigwedge x$ and $u \Vdash \bigwedge y$. Then there are $X_{1}, X_{2} \in \mathcal{S} \Gamma$ (which we hold fixed) such that, for each $x_{1} \in X_{1}$, there is a $t \sqsubseteq s$ such that $t \Vdash \bigwedge x_{1}$ and, for each $x_{2} \in X_{2}$, there is a $u \sqsupseteq s$ such that $s \Vdash \bigwedge x_{2}$. By assumption, there are $x_{1} \in X_{1}$ and $y_{1} \in \mathcal{S} A$ with $x_{1} \subseteq y_{1} \subseteq \bigcup X_{1}$. So, for some $t \sqsubseteq s, t \Vdash \bigwedge x_{1}$. Now let $y-x=\left\{l_{1}, \ldots, l_{n}\right\}$. For each such $l_{i}$, there is a $t_{i} \sqsubseteq s$ such that $t_{i} \Vdash l_{i}$, hence $t \sqcup t_{1} \sqcup \cdots \sqcup t_{n} \Vdash \bigwedge y$ and $t \sqcup t_{1} \sqcup \cdots \sqcup t_{n} \sqsubseteq s$. Similarly, there are $x_{2} \in X_{2}$ and $y_{2} \in \mathcal{S} A$ with $x_{2} \subseteq y_{2} \subseteq \bigcup X_{2}$. So, for some $u \sqsupseteq s, u \Vdash \bigwedge x_{2}$. Since $M$ is non-vacuous, there is a $v$ in $M$ such that $v \Vdash \bigwedge\left(y_{2}-x_{2}\right)$, hence $x_{2} \sqcup v \Vdash \bigwedge y_{2}$, and $s \sqsubseteq\left(x_{2} \sqcup v\right)$. Then lemma 6.4 implies $s \in|A|_{*}^{+}$, and hence $\Gamma \models_{n v *} A$.

§8. Proof Theory. In this section, we set out a proof system corresponding to exact entailment. (We leave a system corresponding to non-vacuous convex exact entailment for future work.) Our proof system defines a derivability relation $\vdash$, holding between a set of sets of formulas and a set of formulas. To avoid an over-abundance of ' $\{$ 's and ' $\}$ 's, we use an alternative notation for writing sequents, as follows:

$$
A_{1}, \ldots, A_{n} ; B_{1}, \ldots, B_{m} ; C_{1}, \ldots, C_{k} \vdash D_{1}, \ldots, D_{i}
$$

Here, we have two premise-grouping pieces of punctuation, ';' and ','. A list ' $A_{1}, \ldots, A_{n}$ ' not broken by a semicolon notates a set of formulas, whereas the concatenation of sets of formulas is notated by the semicolon. So, for example, ' $A ; B ; C$ ' notates the set $\{\{A\},\{B\},\{C\}\}$, whereas ' $A, B, C$ ' notates $\{\{A, B, C\}\}$. Observe that, in this notation, ' $C ; B ; A$ ' and ' $C ; C ; B ; A$ ' also notate the former set, and ' $C, B, A$ ' and ' $C, C, B, A$ ' also notate the latter. (Which set a list notates is not affected by re-ordering or repetition.)

The intuitive interpretation of this notation is that comma-separated formulas are taken conjunctively, whereas semicolon-separated formulas are taken individually. So, the above sequent

$$
A_{1}, \ldots, A_{n} ; B_{1}, \ldots, B_{m} ; C_{1}, \ldots, C_{k} \vdash D_{1}, \ldots, D_{i}
$$

is intended to capture the inference from premises $A_{1} \wedge \cdots \wedge A_{n}, B_{1} \wedge \cdots \wedge B_{m}$, and $C_{1} \wedge \cdots \wedge C_{k}$, to conclusion $D_{1} \wedge \cdots \wedge D_{i}$. Note that this is not the usual multiconclusion sequent, since ' $D_{1}, \ldots, D_{i}$ ' on the right captures the $D$ s conjunctively, not individually.

To preserve the appearance of traditional sequent rules, we will henceforth use upper-case ' $\Gamma$ ', ' $\Delta$ ' as variables for sets of sets of formulas, and use lower-case ' $\gamma$ ', ' $\delta$ ', ' $\xi$ ' as variables for sets of formulas. We then write the general form of a sequent as ' $\Gamma \vdash \gamma$ '. We indicate that $A$ and $B$ (together with the formulas in $\gamma$ ) 
are to be taken conjunctively on the left (or the right) by writing ' $\Gamma$; $\gamma, A, B \vdash \delta$ ' (or ' $\Gamma \vdash \gamma, A, B$ ').

\section{Structural Rules.}

$$
\begin{array}{ccc}
\frac{\Gamma \vdash \delta}{\gamma \vdash \gamma} \text { (ID) } & \frac{\Gamma \vdash \gamma}{\Gamma ; \gamma \vdash \delta} \text { (WEAK) } & \frac{\Gamma \vdash \gamma}{\Gamma \vdash \delta} \\
\frac{\Gamma ; \gamma \vdash \xi}{\Gamma ; \delta \vdash \chi} & \text { (CUT) } \\
\hline
\end{array}
$$

Since this notation for sets is invariant under reordering and repetition, the system automatically has the following left and right exchange and contraction rules, both for the comma (left and right) and the semicolon (on the left only) built in:

$$
\begin{aligned}
& \frac{\Gamma ; \gamma, A, B, \delta \vdash \xi}{\Gamma ; \gamma, B, A, \delta \vdash \xi}(\mathrm{EX}, \mathrm{L}) \frac{\Gamma \vdash \gamma, A, B, \delta}{\Gamma \vdash \gamma, B, A, \delta}(\mathrm{EX}, \mathrm{R}) \frac{\Gamma ; \Delta_{1} ; \Delta_{2} ; \Xi \vdash \gamma}{\Gamma ; \Delta_{2} ; \Delta_{1} ; \Xi \vdash \gamma}(\mathrm{EX} ; \mathrm{L}) \\
& \frac{\Gamma ; \gamma, A, A, \delta \vdash \xi}{\Gamma ; \gamma, A, \delta \vdash \xi}(\mathrm{w}, \mathrm{L}) \quad \frac{\Gamma \vdash \gamma, A, A, \delta}{\Gamma \vdash \gamma, A, \delta}(\mathrm{w}, \mathrm{R}) \quad \frac{\Gamma ; \Delta ; \Delta ; \Xi \vdash \gamma}{\Gamma ; \Delta ; \Xi \vdash \gamma}(\mathrm{w} ; \mathrm{L})
\end{aligned}
$$

Some comments on $(, 1)$ and $(, 2)$ are in order. $(, 1)$ allows premises to be combined with ',' so long as the conclusions are simultaneously combined with ','. Intuitively, (,1) says that, if we can prove $B_{1}$ from $A_{1}$ and $B_{2}$ from $A_{2}$, then we can prove $B_{1} \wedge B_{2}$ from $A_{1} \wedge A_{2}$. (,2) allows us to combine a conclusion, $\gamma$, with any nonempty subset $\delta$ of a conclusion $\delta, \xi$. The intuition here is that, if $\Gamma$ exactly proves $\gamma$, and $\Gamma$ is sufficient for $\delta$ (in the sense that $\Gamma$ exactly proves some superset $\delta, \xi)$, then $\Gamma$ exactly proves $\gamma, \delta$.

These two rules $(, 1)$ and $(, 2)$ perform genuinely distinct duties. If we attempt to combine them into a single rule,

$$
\frac{\Gamma ; \gamma \vdash \xi \quad \Gamma ; \delta \vdash \chi, \sigma}{\Gamma ; \gamma, \delta \vdash \xi, \chi}(\times)
$$

we will be able to prove too much, as the following derivation shows:

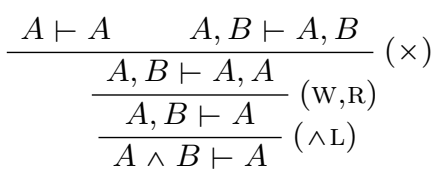

But $A \wedge B \not A$, so $(\times)$ is unsound.

\section{Logical Rules.}

$$
\begin{array}{cc}
\frac{\Gamma ; \gamma, A, B \vdash \delta}{\Gamma ; \gamma, A \wedge B \vdash \delta}(\wedge \mathrm{L}) & \frac{\Gamma \vdash \gamma, A, B}{\Gamma \vdash \gamma, A \wedge B}(\wedge \mathrm{R}) \\
\frac{\Gamma ; \gamma, \neg A, \neg B \vdash \delta}{\Gamma ; \gamma, \neg(A \vee B) \vdash \delta}(\neg \vee \mathrm{L}) & \frac{\Gamma \vdash \gamma, \neg A, \neg B}{\Gamma \vdash \gamma, \neg(A \vee B)}(\neg \vee \mathrm{R}) \\
\frac{\Gamma ; \gamma, A \vdash \xi \quad \Gamma ; \gamma, B \vdash \xi}{\Gamma ; \gamma, A \vee B \vdash \xi}(\vee \mathrm{L}) \frac{\Gamma \vdash \gamma, A / B}{\Gamma \vdash \gamma, A \vee B}(\vee \mathrm{R})
\end{array}
$$




$$
\begin{gathered}
\frac{\Gamma ; \gamma, \neg A \vdash \xi \quad \Gamma ; \gamma, \neg B \vdash \xi}{\Gamma ; \gamma, \neg(A \wedge B) \vdash \xi}(\neg \wedge \mathrm{L}) \quad \frac{\Gamma \vdash \gamma, \neg A / \neg B}{\Gamma \vdash \gamma, \neg(A \wedge B)}(\neg \wedge \mathrm{R}) \\
\frac{\Gamma ; \gamma, A \vdash \delta}{\Gamma ; \gamma, \neg \neg A \vdash \delta}(\neg \neg \mathrm{L}) \quad \frac{\Gamma \vdash \gamma, A}{\Gamma \vdash \gamma, \neg \neg A}(\neg \neg \mathrm{R})
\end{gathered}
$$

A derivation of $\Gamma \vdash \gamma$ is a tree of rule instances, with an instance of (ID) at each leaf and $\Gamma \vdash \gamma$ at the root.

Examples. To illustrate the system, we offer some sample derivations. To derive conjunction introduction:

$$
\frac{A \vdash A \quad B \vdash B}{\frac{A, B \vdash A, B}{A, B \vdash A \wedge B}(\wedge \mathrm{R})}(, 1)
$$

To derive distribution of $\wedge$ over $\vee$ :

$$
\frac{\frac{A \vdash A \quad B \vdash B}{A, B \vdash A, B}(, 1)}{\frac{A \vdash A \quad C \vdash C}{A, B \vdash A \wedge B}(\wedge \mathrm{R})}(\vee \mathrm{R}) \quad \frac{\frac{A \vdash C \vdash A, C}{A, C \vdash A \wedge C}(\wedge \mathrm{R})}{A, C \vdash(A \wedge B) \vee(A \wedge C)}(\vee \mathrm{R})
$$

To derive a disjunction from the corresponding conjunction:

$$
\frac{\frac{A \vdash A}{A \vdash A \vee B}(\vee \mathrm{R}) \quad \frac{B \vdash B}{B \vdash A \vee B}}{\frac{A, B \vdash A \vee B, A \vee B}{A, B \vdash A \vee B}(\mathrm{WR})}(, 1)
$$

§9. Soundness and Completeness. The proof system just presented is sound and complete with respect to exact truthmaking entailment. Let's introduce a bit more terminology to help us through these proofs.

Definition 9.1 (Fit and support). For any set of literals $y$ and any set of sets of literals $X$, we will say that $y$ fits $X$ iff there is some $x \in X$ such that $x \subseteq y \subseteq \bigcup X$. For any set of formulas $\Gamma$ and formula $A$, we say that $\Gamma$ supports $A$ iff, for any $X \in \mathcal{S} \Gamma$, some $y \in \mathcal{S} A$ fits $X$.

The idea here is that $y$ 's fitting $X$ corresponds to $X \vdash y$ (as we show in lemma 9.3 below). We found we were unable to establish soundness in the usual way, via a semantic argument. The proof below goes via characterisation theorem 4.12 from $\S 4$.

Theorem 9.2 (Soundness). For any $\Gamma \subseteq 2^{\mathcal{L}}$, let $\Gamma^{\wedge}=\{\bigwedge \gamma \mid \gamma \in \Gamma\}$. Then $\Gamma \vdash \gamma$ only if $\Gamma^{\wedge} \models \wedge \gamma$.

Proof. Given the characterisation theorem 4.12, it is sufficient to show that $\Gamma^{\wedge}$ supports $\bigwedge \gamma$ whenever $\Gamma \vdash \gamma$. Since this holds trivially for (ID) $(\{A\}$ supports $A$ ), it is sufficient to show that each rule preserves the support relation from upper to lower sequents. We show the cases for our structural rules $(, 1)$ and $(, 2)$, 
and for a sample logical rule, $(\vee \mathrm{L})$. The other structural rules are straightforward, and the cases for the other logical rules are similar to the $(\vee L)$ case.

$(, 1)$ Suppose $(\Gamma \cup\{\gamma\})^{\wedge}$ supports $\bigwedge \xi$ and $(\Gamma \cup\{\delta\})^{\wedge}$ supports $\wedge \chi$. Now consider any $X \in \mathcal{S}\left((\Gamma \cup\{\gamma \cup \delta\})^{\wedge}\right)=\mathcal{S}\left(\Gamma^{\wedge} \cup\{\wedge(\gamma \cup \delta\})\right)$. Then $X=X_{1} \cup\{x\}$ where $X_{1} \in \mathcal{S} \Gamma^{\wedge}$ and $x \in \mathcal{S} \wedge(\gamma \cup \delta)$, hence $x=x_{1} \cup x_{2}$, where $x_{1} \in \mathcal{S} \wedge \gamma$ and $x_{2} \in \mathcal{S} \wedge \delta$. Then $X_{1} \cup\left\{x_{1}\right\} \in \mathcal{S}\left((\Gamma \cup\{\gamma\})^{\wedge}\right)$ and so, by assumption, some $y \in \mathcal{S} \wedge \xi$ fits $X_{1} \cup\left\{x_{1}\right\}$. Similarly, $X_{1} \cup\left\{x_{2}\right\} \in \mathcal{S}\left((\Delta \cup\{\delta\})^{\wedge}\right)$ and so, by assumption, some $z \in \mathcal{S} \wedge \chi$ fits $X_{2} \cup\left\{x_{2}\right\}$. Then there are $u \in X_{1} \cup\left\{x_{1}\right\}$ and $v \in X_{1} \cup\left\{x_{2}\right\}$ such that $u \subseteq y$ and $v \subseteq z$. If $u \in X_{1}$ or $v \in X_{1}$, then either $u \in X$ or $v \in X$. If not, then $u=x_{1}, v=x_{2}$, so $u \cup v \in X$. Either way, we have some $w \in X$ with $w \subseteq y \cup y$. Moreover, $y \subseteq \bigcup X_{1} \cup\left\{x_{1}\right\}$ and $z \subseteq \bigcup X_{1} \cup\left\{x_{2}\right\}$, and so $y \cup z \subseteq \bigcup\left(X_{1} \cup\left\{x_{1}\right\} \cup\left\{x_{2}\right\}\right)=\bigcup X$. So $y \cup z$ fits $X$. Since $y \cup z \in \mathcal{S} \wedge(\xi \cup \chi)$, it follows that $(\Gamma \cup\{\gamma \cup \delta\})^{\wedge}$ supports $\bigwedge(\xi \cup \chi)$.

(,2) Suppose $\Gamma^{\wedge}$ supports both $\bigwedge \gamma$ and $\bigwedge(\delta \cup \xi)$. Now consider any $X \in \mathcal{S} \Gamma^{\wedge}$. By assumption, some $y \in \mathcal{S} \wedge \gamma$ and some $z \in \mathcal{S} \wedge(\delta \cup \xi)$ each fit $X$. Then $z=z_{1} \cup z_{2}$ where $z_{1} \in \mathcal{S} \wedge \delta$ and $z_{2} \in \wedge \xi$, hence $y \cup z_{1} \in \mathcal{S} \wedge(\gamma \cup \delta)$. So there is an $x \in X$ such that $x \subseteq y$, hence $x \subseteq y \cup z_{1}$. Moreover, $y \subseteq \bigcup X$ and $z_{1} \subseteq z \subseteq \bigcup X$, hence $y \cup z_{1} \subseteq \bigcup X$. It follows that $y \cup z_{1}$ fits $X$, and hence that $\Gamma^{\wedge}$ supports $\bigwedge(\gamma \cup \delta)$.

$(\vee \mathrm{L})$ Suppose $(\Gamma \cup\{\gamma \cup\{A\}\})^{\wedge}=\Gamma^{\wedge} \cup\{\wedge \gamma \wedge A\}$ and $(\Gamma \cup\{\gamma \cup\{B\}\})^{\wedge}=$ $\Gamma^{\wedge} \cup\{\wedge \gamma \wedge B\}$ each support $\bigwedge \xi$, and consider any

$$
X \in \mathcal{S}\left((\Gamma \cup\{\gamma \cup\{A \vee B\}\})^{\wedge}\right)=\mathcal{S}\left(\Gamma^{\wedge} \cup\{\bigwedge \gamma \wedge(A \vee B)\}\right) .
$$

Then $X=X_{1} \cup\left\{x_{2} \cup x_{3}\right\}$ where $X_{1} \in \mathcal{S} \Gamma^{\wedge}, x_{2} \in \mathcal{S} \wedge \gamma$, and $x_{3} \in \mathcal{S}(A \vee B)$. Then (i) $x_{3} \in \mathcal{S} A$, (ii) $x_{3} \in \mathcal{S} B$, or (iii) $x_{3}=z_{1} \cup z_{2}$ where $z_{1} \in \mathcal{S} A$ and $z_{2} \in \mathcal{S} B$. If (i) then by assumption, some $y \in \mathcal{S} \wedge \xi$ fits $X_{1} \cup\left\{x_{2} \cup x_{3}\right\}$ and hence $y$ fits $X$. Similarly if (ii). If (iii), then some $y \in \mathcal{S} \wedge \xi$ fits $X_{1} \cup\left\{x_{2} \cup z_{1}\right\}$ and, since $X_{1} \subseteq X$ and $x_{2} \cup z_{1} \subseteq x_{2} \cup x_{3} \in X, y$ also fits $X$. It follows that $(\Gamma \cup\{\gamma \cup\{A \vee B\}\})^{\wedge}$ supports $\bigwedge \xi$.

Now we build up to a completeness proof, by establishing the following lemmas.

Lemma 9.3. If $y$ fits $X$, then $X \vdash y$.

Proof. Suppose $y$ fits $X$. Then by definition, there is some $x \in X$ such that $x \subseteq y \subseteq \bigcup X$ and so, via (ID) and (WEAK), $X \vdash x$. Now let $y-x=\left\{l_{1}, \ldots, l_{n}\right\}$. For each $l_{i}, i \leqslant n$, there is some $z \in X$ such that $l_{i} \in z$, hence $X \vdash z$. So by $n$ applications of $(, 2)$, we get $X \vdash x, l_{1}, \ldots, l_{n}$, which is $X \vdash y$.

Lemma 9.4. If $y \in \mathcal{S} A$ and $y$ fits $X$, then $X \vdash A$.

Proof. By induction on $A$. For the base case, we have $y=\{A\}$ and so, by lemma 9.3, $X \vdash y$ and hence $X \vdash\{A\}$, which we conventionally write as $X \vdash A$. Now assume the result holds for all $B$ less complex than $A$, and consider these cases.

- $A:=A_{1} \wedge A_{2}$. Then $y=y_{1} \cup y_{2}$ where $y_{1} \in \mathcal{S} A_{1}$ and $y_{2} \in \mathcal{S} A_{2}$ and, for some $x \in X$, we have $x \subseteq\left(y_{1} \cup y_{2}\right) \subseteq \bigcup X$. Now let $Y=X-x$, 
$x_{1}=x \cap y_{1}$ and $x_{2}=x \cap y_{2}$. Then $y_{1}$ fits $Y \cup\left\{x_{1}\right\}$ and $y_{2}$ fits $Y \cup\left\{x_{2}\right\}$. So by hypothesis, $Y$; $x_{1} \vdash A_{1}$ and $Y ; x_{2} \vdash A_{2}$. Applying $(, 1)$ and $(\wedge \mathrm{R})$, we derive $Y ; x_{1}, x_{2} \vdash A_{1}, A_{2}$ and then $Y ; x_{1}, x_{2} \vdash A_{1} \wedge A_{2}$. Here, ' $Y ; x_{1}, x_{2}$ ' notates the set

$$
Y \cup\left\{x_{1} \cup x_{2}\right\}=Y \cup\left\{\left(x \cap y_{1}\right) \cup\left(x \cap y_{2}\right)\right\}=Y \cup\{x\}=X
$$

since $x \subseteq\left(y_{1} \cup y_{2}\right)$, and hence $X \vdash A$.

- $A:=A_{1} \vee A_{2}$. Then either $y \in \mathcal{S} A_{1}, y \in \mathcal{S} A_{2}$, or else $y=y_{1} \cup y_{2}$ where $y_{1} \in \mathcal{S} A_{1}$ and $y_{2} \in \mathcal{S} A_{2}$. If $y \in \mathcal{S} A_{1}$ then, by hypothesis, $X \vdash A_{1}$, and by $(\vee \mathrm{R})$, we infer $X \vdash A$. Similarly, if $y \in \mathcal{S} A_{2}$ then $X \vdash A_{2}$ and hence $X \vdash A$. Otherwise, reasoning as in the $\wedge$ case, we infer $X \vdash A_{1}, A_{2}$. Then using ( $\vee \mathrm{R}$ ), we infer $X \vdash A_{1} \vee A_{2}, A_{1} \vee A_{2}$, which (given that contraction is built into our notation) amounts to $X \vdash A$.

- The $A:=\neg\left(A_{1} \vee A_{2}\right)$ and $A:=\neg\left(A_{1} \wedge A_{2}\right)$ cases are similar to the cases above (respectively), and the $A:=\neg \neg A_{1}$ case is trivial.

Lemma 9.5. For any $\Gamma \subseteq 2^{\mathcal{L}}, \gamma \subseteq \mathcal{L}, A$ and $B$ : if $\Gamma ; \gamma, \beta \vdash A$ for each $\beta \in \mathcal{S} B$, then $\Gamma ; \gamma, B \vdash A$.

Proof. By induction on the complexity of $B$. Assume $\Gamma ; \gamma, \beta \vdash A$ for each $\beta \in \mathcal{S} B$. For the base case $B:=p$ or $B:=\neg p$, we have $\mathcal{S} B=\{\{p\}\}$ or $\mathcal{S} B=$ $\{\{\neg p\}\}$, so the result is immediate. For our inductive hypothesis, assume that for all $C$ less complex than $B$, if $\Gamma ; \gamma, \beta \vdash A$ for each $\beta \in \mathcal{S} C$, then $\Delta ; \gamma, C \vdash A$. Now consider these cases.

- $B:=B_{1} \wedge B_{2}$. Then for each $\delta \in \mathcal{S} B, \delta=\delta_{1} \cup \delta_{2}$, where $\delta_{i} \in \mathcal{S} B_{i}$. So by assumption, $\Gamma ; \gamma, \beta_{1}, \beta_{2} \vdash A$ for each $\beta_{i} \in \mathcal{S} B_{i}$ (since $\gamma, \beta_{1}, \beta_{2}$ denotes the set $\left.\gamma \cup \beta_{1} \cup \beta_{2}\right)$. So by hypothesis, $\Gamma ; \gamma, \beta_{1}, B_{2} \vdash A$ for each $\beta_{1} \in \mathcal{S} B_{1}$, and so again by hypothesis, $\Gamma$; $\gamma, B_{1}, B_{2} \vdash A$. Then by $(\wedge \mathrm{L}), \Gamma ; \gamma, B_{1} \wedge B_{2} \vdash A$.

- $B:=B_{1} \vee B_{2}$. Then $\mathcal{S} B_{1} \subseteq \mathcal{S} B$ and $\mathcal{S} B_{2} \subseteq \mathcal{S} B$. So by hypothesis, $\Gamma ; \gamma, B_{1} \vdash A$ and $\Gamma ; \gamma, B_{2} \vdash A$ and, by $(\vee \mathrm{L}), \Gamma ; \gamma, B_{1} \vee B_{2} \vdash A$.

- The $B:=\neg\left(B_{1} \vee B_{2}\right)$ and $B:=\neg\left(B_{1} \wedge B_{2}\right)$ cases are similar to the cases above (respectively), and the $B:=\neg \neg B_{1}$ case is trivial, given that $\mathcal{S}\left(\neg \neg B_{1}\right)=\mathcal{S} B_{1}$.

Theorem 9.6 (Completeness). If $\Gamma \models A$ then $\{\{B\} \mid B \in \Gamma\} \vdash A$.

Proof. Given our compactness theorem 5.2, we may focus on the case in which $\Gamma$ is finite: let $\Gamma=\left\{B_{1}, \ldots, B_{n}\right\}$. So assume $\Gamma \models A$. They by the characterisation theorem, for every $X \in \mathcal{S} \Gamma$, some $y \in \mathcal{S} A$ fits $X$. So by lemma 9.4, $X \vdash A$, for each $X \in \mathcal{S} \Gamma$. Each such $X$ has the form $\left\{\left\{\beta_{1}\right\}, \ldots,\left\{\beta_{n}\right\}\right\}$, where each $\beta_{i} \in \mathcal{S} B_{i}$. In the sequent notation, this is notated: $\beta_{1} ; \ldots ; \beta_{n}$. And by definition of $\mathcal{S} \Gamma$, there is such a set $X$ for each $\beta_{i} \in \mathcal{S} B_{i}$ and each $i \leqslant n$. So we have $\beta_{1} ; \ldots ; \beta_{n} \vdash A$, for each $\beta_{i} \in \mathcal{S} B_{i}$, for each $i \leqslant n$. So by lemma 9.5 (applied $n$ times), we have $B_{1} ; \ldots ; B_{n} \vdash A$, which is equivalent notation for $\left\{\left\{B_{1}\right\}, \ldots,\left\{B_{n}\right\}\right\} \vdash A$.

Theorem 9.7 (Cut-elimination). If $\Gamma \vdash \gamma$ is derivable, then it is derivable without any instances of (CUT). 
Proof. We note that the completeness proof (9.6) does not make use of any instances of (CUT). So the system without (CUT) is sound and complete wrt $\models$, and hence is equivalent to the system with (CUT).

We are unsure of the significance of cut-elimination in this system. In general, the possibility of cut-elimination is important for proof search techniques, since (CUT) is typically the only rule in which a formula vanishes completely from upper to lower sequent (which makes proof search impossible). In the present system, however, the rule $(, 2)$ also contains a formula in an upper sequent which may vanish in the lower sequent. So even cut-free proofs in this system may lack the subformula property. We suspect, but have no proof, that $(, 2)$ is not eliminable from the system.

\section{REFERENCES}

[2016] Kit Fine, Angellic content, Journal of Philosophical Logic, vol. 45 (2016), no. 2, pp. $199-226$.

[2017] - Truthmaker semantics, A companion to the philosophy of language (Bob Hale, Crispin Wright, and Alexander Miller, editors), Blackwell, 2017, pp. 556-577.

[1969] B.C. VAn FraAssen, Facts and tautological entailments, Journal of Philosophy, vol. 66 (1969), no. 15, pp. 477-487.

[2016] ACHILle VARZI, Mereology, The Stanford encyclopedia of philosophy (winter 2016 edition) (Edward N. Zalta, editor), https://plato.stanford.edu/ archives/win2016/entries/mereology/.

DEPARTMENT OF PHILOSOPHY, NEW YORK UNIVERSITY, 5 WASHINGTON PLACE, NEW YORK, NY 10003

E-mail: kit.fine@nyu.edu

DEPARTMENT OF PHILOSOPHY, UNIVERSITY OF NOTTINGHAM, UNIVERSITY PARK, NOTTINGHAM, NG7 2RD

E-mail: mark.jago@nottingham.ac.uk 